\title{
Accelerated 3D whole-brain T1, T2, and proton density mapping: feasibility for clinical glioma MR imaging
}

\author{
Carolin M. Pirkl ${ }^{1,2}$ (1) - Laura Nunez-Gonzalez ${ }^{3}$. Florian Kofler ${ }^{1} \cdot$ Sebastian Endt ${ }^{1,2} \cdot$ Lioba Grundl $^{4}$. \\ Mohammad Golbabaee ${ }^{5}$. Pedro A. Gómez ${ }^{1}$ • Matteo Cencini ${ }^{6,7}$. Guido Buonincontri ${ }^{6,7}$ • Rolf F. Schulte ${ }^{2}$. \\ Marion Smits ${ }^{3} \cdot$ Benedikt Wiestler $^{4,8} \cdot$ Bjoern H. Menze $^{1,9} \cdot$ Marion I. Menzel $^{2,10}$ • Juan A. Hernandez-Tamames ${ }^{3}$
}

Received: 1 February 2021 / Accepted: 28 March 2021 / Published online: 9 April 2021

(C) The Author(s) 2021

\begin{abstract}
Purpose Advanced MRI-based biomarkers offer comprehensive and quantitative information for the evaluation and characterization of brain tumors. In this study, we report initial clinical experience in routine glioma imaging with a novel, fully 3D multiparametric quantitative transient-state imaging (QTI) method for tissue characterization based on T1 and T2 values.

Methods To demonstrate the viability of the proposed 3D QTI technique, nine glioma patients (grade II-IV), with a variety of disease states and treatment histories, were included in this study. First, we investigated the feasibility of 3D QTI (6:25 min scan time) for its use in clinical routine imaging, focusing on image reconstruction, parameter estimation, and contrast-weighted image synthesis. Second, for an initial assessment of 3D QTI-based quantitative MR biomarkers, we performed a ROI-based analysis to characterize $\mathrm{T} 1$ and $\mathrm{T} 2$ components in tumor and peritumoral tissue.

Results The 3D acquisition combined with a compressed sensing reconstruction and neural network-based parameter inference produced parametric maps with high isotropic resolution $\left(1.125 \times 1.125 \times 1.125 \mathrm{~mm}^{3}\right.$ voxel size $)$ and whole-brain coverage $(22.5$ $\times 22.5 \times 22.5 \mathrm{~cm}^{3} \mathrm{FOV}$ ), enabling the synthesis of clinically relevant T1-weighted, T2-weighted, and FLAIR contrasts without any extra scan time. Our study revealed increased $\mathrm{T} 1$ and $\mathrm{T} 2$ values in tumor and peritumoral regions compared to contralateral white matter, good agreement with healthy volunteer data, and high inter-subject consistency.

Conclusion 3D QTI demonstrated comprehensive tissue assessment of tumor substructures captured in T1 and T2 parameters. Aiming for fast acquisition of quantitative MR biomarkers, 3D QTI has potential to improve disease characterization in brain tumor patients under tight clinical time-constraints.
\end{abstract}

Keywords MRI $\cdot$ Image-based biomarkers $\cdot$ Multiparametric imaging $\cdot$ Glioma imaging $\cdot$ Neural networks

Bjoern H. Menze, Marion I. Menzel and Juan A. Hernandez-Tamames contributed equally to this work.

Carolin M. Pirkl

carolin.pirkl@tum.de

1 Department of Computer Science, Technical University of Munich, Munich, Germany

2 GE Healthcare, Munich, Germany

3 Radiology \& Nuclear Medicine, Erasmus MC, University Medical Center, Rotterdam, Netherlands

4 Department of Neuroradiology, Klinikum rechts der Isar, Munich, Germany
Department of Computer Science, University of Bath, Bath, UK

6 Fondazione Imago7, Pisa, Italy

7 IRCCS Fondazione Stella Maris, Pisa, Italy

8 TranslaTUM - Central Institute for Translational Cancer Research, Munich, Germany

9 Department of Quantitative Biomedicine, University of Zurich, Zurich, Switzerland

10 Department of Physics, Technical University of Munich, Munich, Germany 


\section{Introduction}

Gliomas are the most frequent primary brain tumors in adults. This diverse group of brain tumors comprises glioblastomas, astrocytomas, oligodendrogliomas, and ependymomas [1]. Although there have been great advances in glioma research, and treatment continues to evolve with new methods and strategies, gliomas remain a disease with poor prognosis [2]. State-of-the-art glioma treatment includes a multi-disciplinary approach, combining surgical resection, chemotherapy, and radiation therapy [3]. Treatment strategy and prognosis for each individual case depend on tumor grade, which is defined upon histopathologic appearance and molecular features according to the 2016 WHO criteria [4]. High-grade gliomas (grade IV), so called glioblastomas, are aggressive, fastgrowing tumor types that require immediate treatment. For lower-grade gliomas (grades II, III), including various types of astrocytic, oligodendroglial, and ependymal tumors, extensive treatment is often delayed as long as possible [5].

For all types of gliomas, comprehensive multimodal neuroimaging is fundamental for disease characterization $[6,7]$. It also guides the individualized therapy planning and is required to monitor treatment response and progression of the disease. Here, MRI has become the key diagnostic measure for the evaluation and characterization of brain tumors: while the multitude of image contrasts of conventional structural MRI allows for better detection of tumor-infiltrated areas, advanced image-based physiologic and molecular biomarkers have been demonstrated to offer comprehensive and quantitative information about the biological characteristics of tumor types and tumor substructures [8]. For the ultimate goal of an as-precise-as-possible therapy [9], quantitative MRI can therefore provide versatile tissue characterization [10]. This in turn is essential to better comprehend the complex proliferative and invasive behavior, to identify and describe structures of interest, such as enhancing tumor structures, or critical thresholds in a reliable and reproducible way to better predict therapy response and treatment outcomes.

Usually, long acquisition times of such conventional quantitative MR techniques, however, hinder their adoption into clinical practice. Routine imaging protocols therefore rely on mainly qualitative information so far. Also, visual inspection and qualitative interpretation are dominating clinical MRI-based diagnosis because the analysis of complex multi-parametric, multimodal, and even multi-temporal image data sets remains a major challenge. These issues, together with the lack of MRI protocol standardization [11], hamper a reliable identification of tumor substructures, render an exact quantification of infiltration patterns impossible, and complicate monitoring of treatment response in follow-up examinations.

To meet the clinical need for fast acquisition of quantitative MR biomarkers, advanced multiparametric MRI schemes have been proposed, offering reproducible and accurate diagnostic information, which is less affected by system and interpretation biases [12-16]. They all share the common goal of revealing clinically relevant tissue characteristics, which are not appropriately captured in standard qualitative MRI, with clinically practicable scan times.

Aiming for joint $\mathrm{T} 1$ and $\mathrm{T} 2$ mapping, different acquisition and data processing strategies based on (undersampled) $\mathrm{k}$ space data are used to achieve optimal multiparametric estimation $[12-15,17]$. In this work, we present the feasibility of a novel, fully $3 \mathrm{D}$ multiparametric quantitative transient-state imaging (QTI) technique [18] for simultaneous mapping of $\mathrm{T} 1$ and $\mathrm{T} 2$ relaxation times and relative proton density (PD) for use in clinical routine glioma imaging. With 3D QTI, we demonstrate a 3D acquisition with high isotropic resolution that allows us to go beyond the resolution of other recently presented quantitative MRI methods based on 2D (multi-)slice acquisitions. We pursue a conceptionally different approach compared to steady-state magnetization techniques and acquire the signal evolution in the transient state. In

Table 1 Patient demographics, diagnoses, and treatment histories

\begin{tabular}{lllll}
\hline Patient ID & Age & Gender & Diagnosis & Treatment \\
\hline 1 & $69 \mathrm{y}$ & $\mathrm{f}$ & Giant cell glioblastoma, IDH wild type & Resection, radiation therapy, chemotherapy \\
2 & $63 \mathrm{y}$ & $\mathrm{m}$ & Anaplastic astrocytoma (WHO grade III) & Chemotherapy \\
3 & $49 \mathrm{y}$ & $\mathrm{m}$ & Glioblastoma, IDH wild type & Chemotherapy \\
4 & $69 \mathrm{y}$ & $\mathrm{m}$ & Glioblastoma & Resection, chemotherapy \\
5 & $63 \mathrm{y}$ & $\mathrm{m}$ & Transitional cell oligodendroglioma (WHO grade II) & Resection, radiation therapy \\
6 & $52 \mathrm{y}$ & $\mathrm{f}$ & Glioblastoma & Resection, radiation therapy, chemotherapy \\
7 & $50 \mathrm{y}$ & $\mathrm{m}$ & Oligodendroglioma (WHO grade II) & Resection, radiation therapy, chemotherapy \\
8 & $58 \mathrm{y}$ & $\mathrm{f}$ & Anaplastic oligodendroglioma, IDH mutant and 1p/19q co-deleted & Resection, chemotherapy \\
9 & $25 \mathrm{y}$ & $\mathrm{f}$ & Low-grade astrocytoma & Resection, chemotherapy \\
\hline
\end{tabular}


Table 2 MR sequence parameters

\begin{tabular}{|c|c|c|c|c|c|}
\hline & 3D QTI & $\begin{array}{l}\text { T1-weighted FSPGR } \\
\text { (T1w) }\end{array}$ & $\begin{array}{l}\text { Gd-enhanced T1-weighted } \\
\text { FSPGR (T1c) }\end{array}$ & $\begin{array}{l}\text { T2-weighted } \\
\text { PROPELLER (T2w) }\end{array}$ & $\begin{array}{l}\text { CUBE FLAIR } \\
\text { (FLAIR) }\end{array}$ \\
\hline Acquisition & $3 \mathrm{D}$ & $3 \mathrm{D}$ & $3 \mathrm{D}$ & $2 \mathrm{D}$ & $3 \mathrm{D}$ \\
\hline $\begin{array}{l}\text { Native resolution } \\
\qquad\left(\mathrm{mm}^{3}\right)\end{array}$ & $\begin{array}{l}1.125 \times 1.125 \times \\
1.125\end{array}$ & $0.47 \times 0.47 \times 0.8$ & $0.47 \times 0.47 \times 0.8$ & $0.5 \times 0.5 \times 3.3$ & $0.8 \times 0.47 \times 0.47$ \\
\hline Matrix size & $200 \times 200 \times 200$ & $512 \times 512 \times 212$ & $512 \times 512 \times 212$ & $512 \times 512 \times 46$ & $192 \times 512 \times 512$ \\
\hline Field of view $\left(\mathrm{mm}^{3}\right)$ & $225 \times 225 \times 225$ & $240 \times 240 \times 170$ & $240 \times 240 \times 170$ & $260 \times 260 \times 152$ & $154 \times 240 \times 240$ \\
\hline Slices & - & - & - & 46 & - \\
\hline $\begin{array}{l}\text { Native slice thickness } \\
\quad(\mathrm{mm})\end{array}$ & - & - & - & 3.0 & - \\
\hline $\mathrm{TE}(\mathrm{ms})$ & 1.8 & 2.1 & 2.1 & 120.7 & 92 \\
\hline TR (ms) & 7.8 & 4.6 & 7.1 & 5751 & 5002 \\
\hline TI (ms) & 18 & - & - & - & 1701 \\
\hline$a\left(^{\circ}\right)$ & $0.8 \leq a \leq 70$ & 12 & 12 & 160 & 90 \\
\hline $\begin{array}{l}\text { Acquisition time } \\
\text { (min) }\end{array}$ & $6: 25$ & $1: 54$ & $4: 43$ & $5: 15$ & $4: 48$ \\
\hline
\end{tabular}

contrast to Cartesian readout schemes, the combination of efficient spiral k-space (under-)sampling with transientstate imaging in 3D QTI constitutes an attractive candidate for fast multiparametric MRI under tight clinical time constraints.

As a form of clinical stress test for 3D QTI, we chose a variety of glioma patients (grades II-IV) with heterogenous disease states and treatment histories to demonstrate the viability of this technique. In this study, we focus on two main aspects:

I. Initial clinical experience with 3D QTI: We demonstrate the feasibility of this fast, multiparametric sequence with whole-brain coverage and high isotropic resolution for being used in routine brain tumor imaging protocols. We examine the applicability of 3D QTI with its clinically relevant scan time of 6:25 min, focusing on its image reconstruction and parameter estimation approaches. Based on the quantitative parameter maps, we synthesize qualitative image contrasts and explore their clinical relevance. We also assess the behavior of the 3D QTI scheme in the presence of patient movement, i.e., rigid head motion and non-rigid physiological motion.

II. Application to quantitative characterization of tumor substructures: We identify tumor tissue heterogeneity that is captured by $\mathrm{T} 1$ and $\mathrm{T} 2$ values to offer comprehensive tissue assessment of tumor substructures and quantifiable differentiation of healthy tissue. We therefore characterize T1 and T2 components in a variety of glioma patients with different disease stages and treatment histories. We aim to gain insights into potential benefits of 3D QTI in cases where pseudo-regression, pseudo-therapy response, or radiation-induced necrosis complicate follow-up assessments [19].

\section{Materials and methods}

\section{Subjects}

Within the course of this study, we collected MR data and respective demographic and clinical data from nine glioma patients who had been scheduled for follow-up clinical imaging (Table 1). The study included a variety of patients who were initially diagnosed with glioblastoma (anaplastic and low-grade), astrocytoma (transitional cell and anaplastic), or oligodendroglioma. Prior treatment strategies cover surgical resection, chemotherapy, radiation therapy, or a combination thereof.

\section{MR imaging}

\section{Clinical contrast-weighted MRI}

All MRI data were acquired on a 3T MR750 system (GE Healthcare, Milwaukee, WI) using a 16-channel head, neck, and spine array coil. The multimodal MRI protocol included a pre-contrast T1-weighted fast spoiled gradient echo (FSPGR) sequence (T1w), a T2-weighted PROPELLER sequence (T2w), and a fluid-attenuated inversion recovery (FLAIR) sequence which were followed by a gadolinium (Gd)-enhanced T1-weighted FSPGR sequence (T1c). All imaging parameters are shown in Table 2.

\section{D QTI acquisition and reconstruction}

In addition to the clinical sequences, and before contrast agent administration, the patients were scanned with the proposed 3D QTI acquisition with an inversion time TI $=18 \mathrm{~ms}$, repetition time $\mathrm{TR}=7.8 \mathrm{~ms}$, and echo time $\mathrm{TE}=1.8 \mathrm{~ms}$. Flip angles $\left(0.8^{\circ} \leq a \leq 70^{\circ}\right)$ follow a ramp-up/ramp-down pattern, 


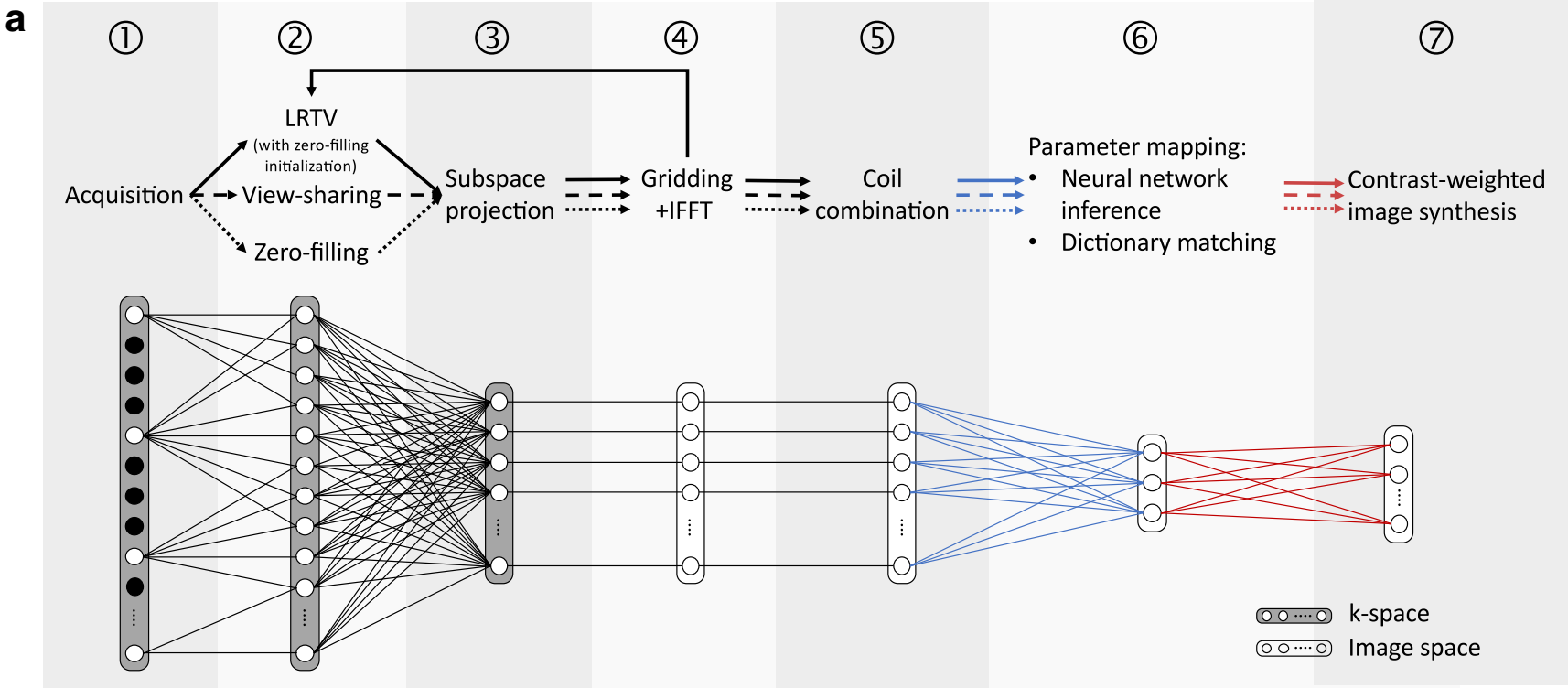

b

(6)

Parameter mapping: Neural network inference

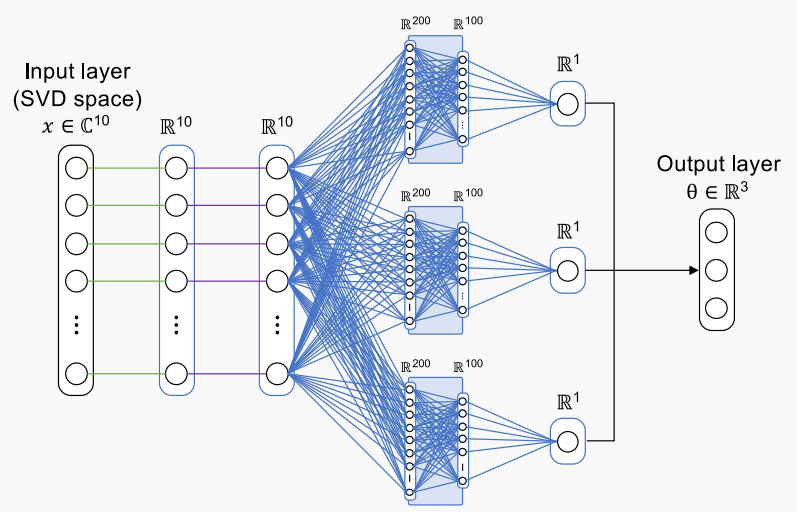

Fig. 1 3D QTI data processing. a Reconstruction and processing. After acquisition (1), raw k-space data is processed via naïve zero-filling (dotted line), k-space weighted view-sharing (dashed line), or a compressed sensing LRTV technique (solid line). All methods in (2) are followed by dimensionality reduction via SVD subspace projection in the time domain (3), gridding onto a Cartesian grid followed by a 3D IFFT (4), and coil sensitivity estimation and combination (5)). The reconstructed image series are then fed into a neural network or are matched to a precomputed dictionary to output parametric maps of $\mathrm{T} 1, \mathrm{~T} 2$ and $\mathrm{PD}$ (6). We then

comprising 880 repetitions. Highly undersampled k-space data (undersampling factor of 628 for each of the $8803 \mathrm{D} \mathrm{k-}$ space volumes) is acquired in the transient state $[16,20]$ using a spiral readout $\left(22.5 \times 22.5 \times 22.5 \mathrm{~cm}^{3} \mathrm{FOV}, 1.125 \times 1.125 \times\right.$ $1.125 \mathrm{~mm}^{3}$ isotropic voxel size) with in-plane and spherical rotations to achieve full 3D coverage. The total scan time of the 3D QTI acquisition was 6:25 min.

3D QTI data was reconstructed using a compressed sensing (CS) approach with joint spatial and temporal synthesize clinical image contrasts using the parametric maps (7)). b Neural network architecture for parameter inference. The model receives the complex, voxel-wise signal in SVD subspace $x$ and infers the underlying tissue parameter vector $\boldsymbol{\theta}$ with T1, T2, and a PD-related scaling factor. The input signal $\boldsymbol{x}$ is phase-aligned (green lines) to transfer the complex into real-valued signal, followed by a normalization layer (purple lines). The model then divides into separate pathways, each with three ReLU-activated hidden layers and 200, 100, and one node, to eventually yield the concatenated parametric output vector $\theta$

regularizations, referred to as low-rank and total-variation (LRTV) method [21]. To demonstrate the anti-aliasing that is achieved by this iterative $\mathrm{k}$-space processing, we compared this reconstruction to naïve zero-filling and $\mathrm{k}$-space weighted view-sharing [22]. Figure 1 a schematically shows the 3D QTI reconstruction pipeline with these three k-space processing alternatives in step (2). In all cases, we applied dimensionality reduction via SVD subspace projection (step (3) to compress the full temporal signal evolution to its first 


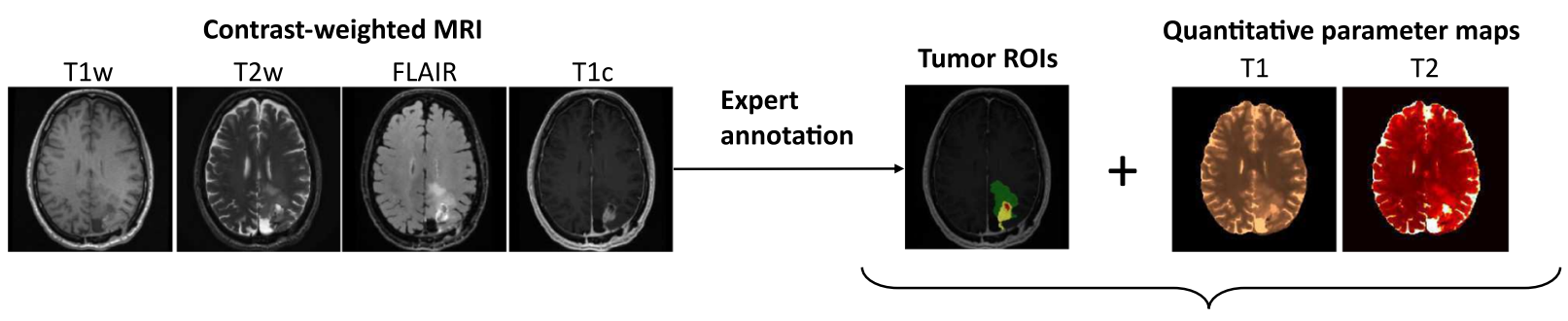

T1-T2 distributions
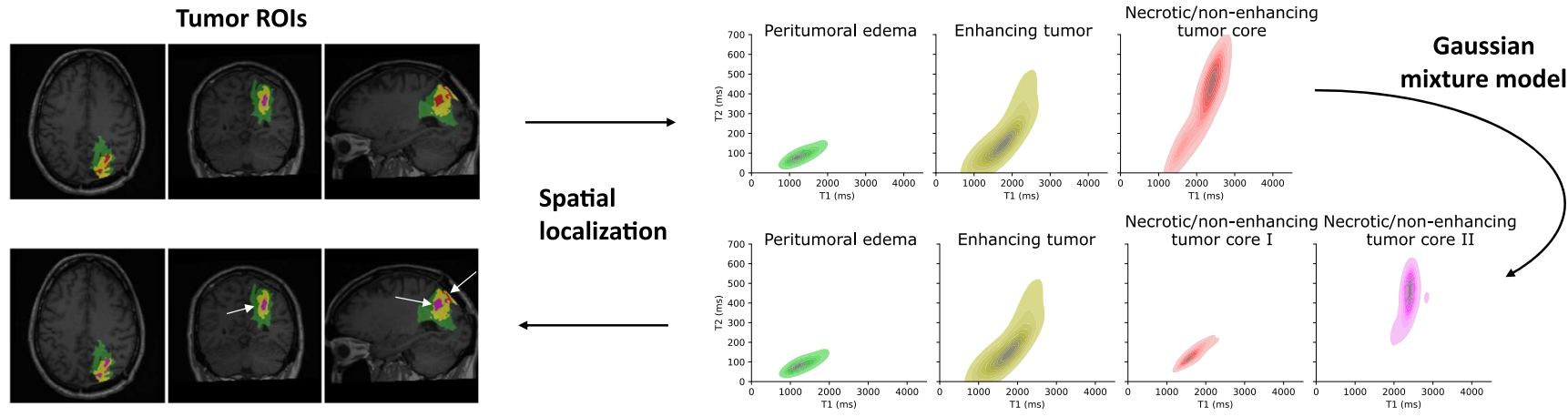

Fig. 2 Application to quantitative characterization of tumor substructures. For quantitative analysis of tumor substructures, intratumoral structures, i.e., peritumoral edema, necrotic/non-enhancing tumor core, and enhancing tumor, were annotated by a trained expert based on the clinical contrast-weighted MR data. Voxel-wise T1-T2

distributions were then derived for the individual ROIs. Using a Gaussian mixture model, we explored whether we can identify the two voxel classes that are apparent in the T1-T2 space in necrotic/nonenhancing tumor areas, which were then mapped back to the image space

ten singular images. SVD projection was followed by gridding onto a Cartesian grid using gpuNUFFT [23] and 3D inverse fast Fourier transform (IFFT, step (4)). In step (5), coil sensitivity maps were computed using adaptive coil combination [24].

For parameter mapping in step (6), the reconstructed, complex SVD images were fed into a compact multi-path neural network for voxel-wise T1, T2, and PD inference, which has shown to be a time and memory-efficient alternative to conventional dictionary matching $[25,26]$. Note that neural network inference and dictionary matching can be applied independent of the previously performed k-space processing.

The proposed neural network architecture, as depicted in Fig. $1 \mathrm{~b}$, receives the first ten singular components of the SVD compressed QTI signal $\mathbf{x}$ as input and outputs the underlying tissue parameters $\mathrm{T} 1, \mathrm{~T} 2$, and a PD-related scaling factor, comprised in the output vector $\theta$, with the final PD estimate $\mathrm{PD}=\frac{\|\mathbf{x}\|_{2}}{\theta_{3}}$. The latent-space input signal is phase-aligned [18], transferring the complex into a real-valued signal, and normalized in the subsequent layer. The model then splits into separate pathways, each consisting of three hidden layers with rectified linear unit (ReLU) activations and 200, 100, and one node, to eventually form the concatenated parametric output $\theta$.

To train the neural network, we generated a dataset of synthetic QTI signals for $10 \mathrm{~ms} \leq \mathrm{T} 1 \leq 5000 \mathrm{~ms}$ and $10 \mathrm{~ms} \leq \mathrm{T} 2 \leq$ $2000 \mathrm{~ms}$ using the extended phase graphs formalism [27]. T1 values were sampled in steps of $10 \mathrm{~ms}$ for $10 \mathrm{~ms} \leq \mathrm{T} 1 \leq 2000 \mathrm{~ms}$ and in steps of $100 \mathrm{~ms}$ for $2100 \mathrm{~ms} \leq \mathrm{T} 1 \leq 5000 \mathrm{~ms}$. T2 values were increased in steps of $5 \mathrm{~ms}$ for $10 \mathrm{~ms} \leq \mathrm{T} 2 \leq 300 \mathrm{~ms}$ and in steps of $10 \mathrm{~ms}$ for $310 \mathrm{~ms} \leq \mathrm{T} 2 \leq 2000 \mathrm{~ms}$. The dataset was also used to obtain a dictionary matching reference. For model training, we used $80 \%$ of the samples in the simulated dataset and added white complex Gaussian noise to the generated signal time-series. The network was trained for a maximum of 1000 epochs with mean absolute percentage error loss and stochastic gradient descent optimization with a learning rate of $1 \mathrm{e}-4$ and a dropout rate of 0.8 . We kept the model state that achieved the best validation loss for the remaining $20 \%$ of the signals.

With the obtained T1, T2, and PD estimates, we generated synthetic T1-weighted, T2-weighted, and T2-weighted FLAIR image contrasts by applying the respective voxelwise signal equations to the estimated parameter maps, as motivated by $[18,28]$. To assess the quality of the synthetic images, we evaluated them against the corresponding acquisitions in the clinical protocol.

\section{Annotation and quantitative analysis of tumor substructures}

For quantitative analysis of tumor substructures in terms of T1 and T2 parameter values, as schematically shown in Fig. 2, intra-tumoral structures - peritumoral edema, necrotic/nonenhancing tumor core, and enhancing tumor core-were annotated by a trained radiologist using ITK SNAP [29] in each 
Fig. 3 Contrast-weighted image synthesis for a representative patient case. From the T1, T2, and PD maps, we produce clinically relevant, fully $3 \mathrm{D}$ qualitative image information with high isotropic resolution and without additional scan time. As seen from the axial views and the histogram-based comparison considering the whole image volumes, synthetic T1-weighted, T2weighted, and FLAIR MRI contrasts correspond to the clinical reference acquisitions.

Corresponding sagittal and coronal views are shown in Fig. 11
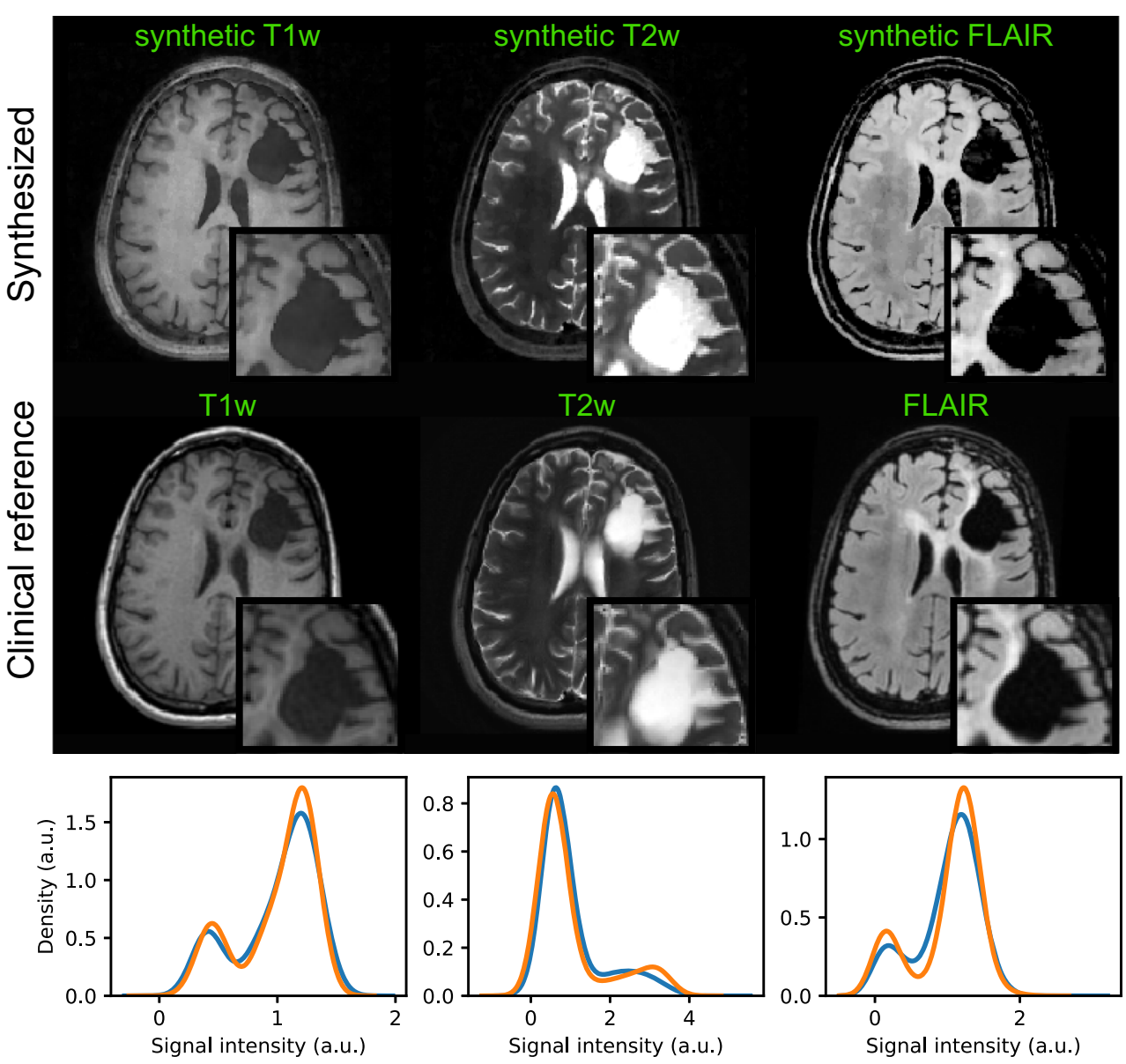

Synthesized Reference patient dataset based on the clinical contrast-weighted MRI data. The T1-weighted, T2-weighted, FLAIR, and Gdenhanced T1-weighted images were therefore transformed into the 3D QTI image space using ANTs [30].

In addition to the tumor annotations, we obtained white matter (WM) and gray matter (GM) tissue segmentations using the FSL FAST algorithm [31], which we applied to the synthesized T1-weighted image data. For the glioma patients, we only considered contralateral WM and GM. If the tumor or its peritumoral tissue affected both hemispheres, normal-appearing WM and GM regions were delineated in the hemisphere with less tumor-affected volume.

Voxel-wise T1-T2 distributions were then identified for individual ROIs. We also compared ROI-based mean T1 and T2 among individual patients. For an initial attempt to explore whether the obtained T1 and T2 information allows us to go beyond the manual segmentation, we fitted a Gaussian mixture model to the T1-T2 space of all necrotic/non-enhancing voxels in the cohort to understand whether we can identify the two apparent tissue types, which we attribute to solid tumor (necrotic/nonenhancing tumor core I) and fluidic tissue voxels (necrotic/nonenhancing tumor core II) therein. The fitted model was then applied to the individual patient datasets to disentangle the voxels in the necrotic/non-enhancing ROI into two classes. The thereby obtained subclassification of necrotic/non-enhancing voxels was then mapped back to the anatomical context to complement the clinical baseline labeling based on qualitative visual MRI contrasts

\section{Results}

We first present and evaluate the 3D QTI method with its modular data processing pipeline. Accuracy and precision of acquisition and reconstruction elements of 3D QTI were evaluated in $[18,21,32]$. Focus of the work presented here is to assess the applicability of 3D QTI for clinical routine imaging in terms of reconstruction performance and image quality of the multiparametric maps. We then use the multiparametric output of 3D QTI to identify tumor tissue heterogeneity and to understand whether this allows a quantifiable differentiation of tumor substructures and healthy tissue in cancer patients. 
Fig. 4 Sensitivity to rigid head motion. Profuse head motion can affect image acquisition in the transient-state, which leads to image degradation in the parametric maps (a) and the synthetic image contrasts (b) compared to the clinical contrastweighted acquisitions. The postcontrast T1-weighted MRI indicates that state-of-the-art conventional MRI cannot fully recoup the pronounced head motion in this case. Corresponding sagittal and coronal views are shown in Fig. 12 a
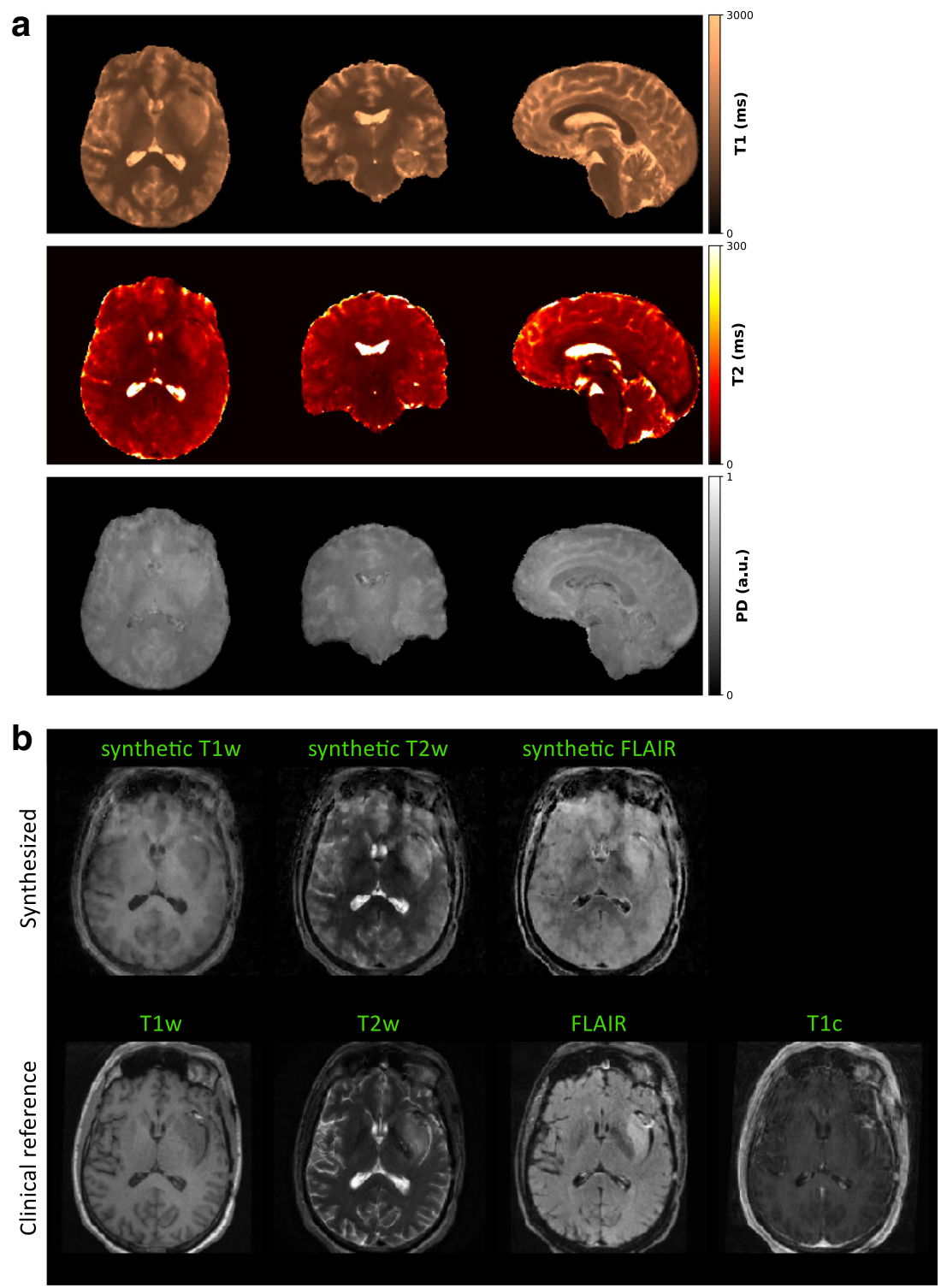

\section{Initial clinical experience with 3D QTI}

Figure 9 illustrates the isotropic 3D maps of T1, T2, and PD that we obtained from the three reconstruction modules with subsequent dictionary matching for a representative patient case and a healthy volunteer. All subsequent results in this study rely on LRTV-based image reconstruction. Figure 10 shows parameter quantification results obtained via neural network-based inference and conventional dictionary matching for a representative patient dataset and a healthy volunteer. From the estimated T1, T2, and PD maps, we synthesized common MRI contrasts using the respective MR signal equations. In Fig. 3 and Fig. 11, we compare the synthetic images for T1-weighted, T2-weighted, and FLAIR image contrasts to the images that were acquired as part of the clinical protocols. As part of the sensitivity analysis towards rigid head movements and physiological motion, Fig. 4 and Fig. 12 show an exemplary case of pronounced patient movement, where motion-related artifacts degrade the image quality in the parametric maps and therefore affect the synthesized MRI contrasts. From Fig. 5 and Fig. 12, we observe how physiological motion, such as blood flow and CSF pulsation effects, impact parameter quantification and subsequent contrast-weighted image synthesis.

\section{Application to quantitative characterization of tumor substructures}

Figure 6 gives an overview of the tumor ROI annotations together with the clinical contrast-weighted MRIs and the obtained T1 and T2 maps for all patients. In all cases, tumor core (red annotation) and peritumoral edema regions (green 
Fig. 5 Sensitivity to physiological motion. Pulsating blood flow and thereby induced pulsation of the cerebrospinal fluid (CSF) can impact the T2 estimation (a) and subsequent synthesis of T2-weighted image contrasts (b) as observed in large vessels and in regions with high CSF pulsation, e.g., along the brainstem (white arrows) a

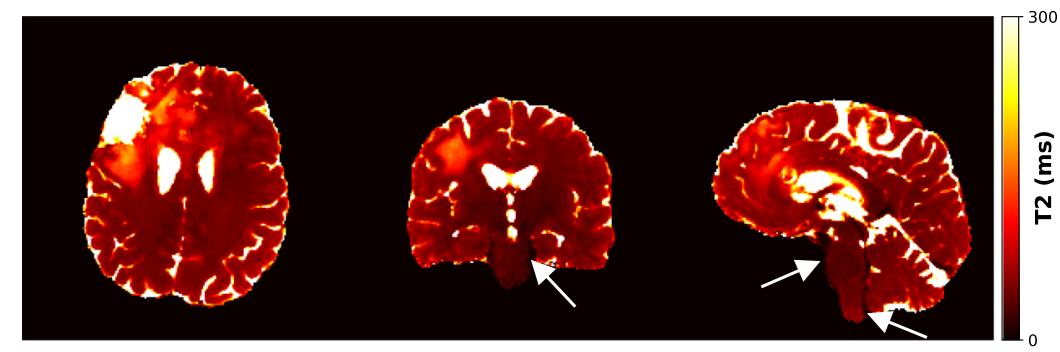

b

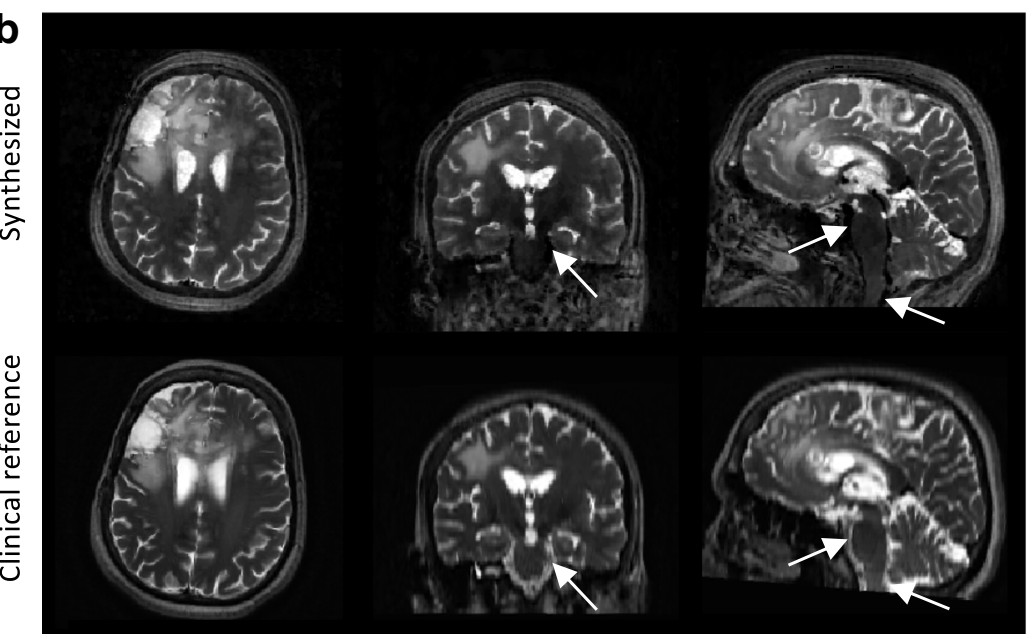

annotation) appear hyperintense on the conventional T2weighted and FLAIR images and hypointense on T1weighted images, relative to normal appearing tissue. T1 and $\mathrm{T} 2$ values obtained in these regions are higher compared to healthy tissue areas. Post-contrast T1-weighted images of patients 1 to 4 and 6 additionally identify areas with Gd enhancement (yellow annotation). Patients 3, 4, and 6 are cases with clearly visible gross tumor volumes. For patients 1 and 8, contrast-weighted MRIs do not indicate tumor relapse around the resection cavities. In case of patient 8 , there are small tumor-suspected findings in the corpus callosum and the left anterior horn of the lateral ventricle without hyperintensities in the post-contrast T1-weighted MRI. In case of patient 1, the post-contrast MRI reveals small findings that are positive for Gd enhancement. For patient 2, there are small, discrete areas of Gd enhancement, which might indicate diffuse tumor growth, surrounded by edema. For patient case 5, 7, and 9, there is no clear sign for tumor reoccurrence after resection. In these cases, tumor ROIs only comprise areas of peritumoral edema and gliosis.

Figure 7 illustrates exemplary results for patient case 4 and 6 that we obtained from the Gaussian mixture model, when trained on all voxels in the patients' dataset labeled as necrotic/non-enhancing tumor tissue. The two voxel types, i.e., necrotic/non-enhancing tumor I (red) and necrotic/nonenhancing tumor core II (magenta) that are identified in the T1-T2 parameter space were projected back into the anatomical context to complement the manual ROI segmentation.
Figure 8 and Table 3 quantitatively summarize the ROIbased analysis of tumor substructures. Quantitative T1 and T2 mapping results obtained in a healthy volunteer are reported in Table 4.

\section{Discussion}

This study aimed to evaluate the feasibility of 3D quantitative transient-state imaging (QTI) for clinical imaging of glioma patients. First, we demonstrated a feasibility analysis of QTIbased, fully 3D multiparametric MRI for integration into stateof-the-art clinical routine brain tumor protocols with strict requirements regarding acquisition times and robustness. Second, we showed that 3D QTI offers comprehensive characterization of both healthy and diseased tissue in a variety of brain tumor patients. Despite the heterogeneity of the patient cohort, this approach captures tissue heterogeneity in tumor substructures based on quantifiable $\mathrm{T} 1$ and $\mathrm{T} 2$ parameters.

\section{Initial clinical experience with 3D QTI}

\section{Multiparametric mapping}

Initial experience with 3D QTI in glioma patients demonstrated fully quantitative, multiparametric MR mapping with high isotropic resolution and an acquisition time of 6:25 $\mathrm{min}$ that make it feasible for use under tight clinical time 
constraints. We observed that parameter quantification is consistent across the different reconstruction approaches provided by the 3D QTI pipeline, i.e., zero-filling, viewsharing, and LRTV methods (Fig. 9). This is in correspondence with previous study results $[18,21]$. View-sharing and LRTV reconstruction can improve spatial consistency in the reconstructed SVD image series compared to naïve zerofilling as reflected in an increased image quality of the inferred parameter maps. The iterative LRTV reconstruction with joint spatio-temporal regularization achieves best suppression of aliasing artifacts. It provides best image quality and maintains clinically important tissue changes and critical tissue interfaces within tumor and peritumoral regions. That is, complementing the fast 3D QTI acquisition with a compressed sensing reconstruction with joint spatiotemporal regularization has demonstrated convincing capacities to suppress aliasing artifacts, producing highquality parametric maps. In 3D QTI, acquisition and reconstruction are well aligned, allowing to successfully mitigate inherent practical concerns of spiral sampling such as gradient imperfections or spiral artifacts due to a massively undersampled k-space. As such, we take advantage of the high scanning efficiency of spiral trajectories and use it in clinical routine imaging, as an alternative to the prevalent Cartesian readout schemes. Based on the initial results presented here, we are confident that we can further advance 3D QTI, e.g., to smaller voxel sizes or faster scanning times.

Comparison of neural network-based inference and dictionary matching (Fig. 10) showed that both approaches produce T1, T2, and PD maps that are largely consistent in terms of quantification accuracy and image quality as previously shown by Gómez et al. [18]. As such, the neural network provides high-resolution maps with quantification accuracy and image quality comparable to dictionary matching. This is observed for healthy tissue, i.e., of the volunteer scan and normal-appearing tissue regions in glioma patients, as well as in tumor regions with alterations of the tissue microstructure.

With the combination of the CS-based LRTV reconstruction and the neural network-based parameter inference, we therefore present a memory-efficient, dictionary-free reconstruction pipeline.

\section{Synthetic MRI}

Given the premise of an as-short-as-possible imaging protocol, we have shown that contrast-weighted image synthesis based on the multiparametric 3D QTI output can produce fully 3D, high-quality, and clinically relevant qualitative information without prolonging the scan session (Fig. 3). As such, it offers an attractive feature with the potential to replace conventional contrast-weighted acquisitions, including T1weighted, T2-weighted, and FLAIR contrasts, to potentially reduce the required scan times of routine brain imaging protocols (Table 2). Note that the synthetic contrast-weighted MRIs are naturally obtained in the same image space. That is, expensive processing, i.e., co-registration and resampling of image volumes, which is generally a key requirement in multimodal studies in order to homogenize the individual datasets, becomes redundant. So far, image synthesis based on $\mathrm{T} 1, \mathrm{~T} 2$, and PD estimates is confined to native, i.e., precontrast image contrasts, and can therefore not replace Gdenhanced acquisitions yet. In light of the ongoing research efforts to reduce the use of contrast agents to an absolute minimum, there have been initial studies suggesting that T1relaxometry can potentially provide equivalent insights into tissue characteristics as qualitative post-contrast information [33]. However, based on our results, we cannot draw such conclusions solely based on native $\mathrm{T} 1$ and $\mathrm{T} 2$ parameters, i.e., without the inclusion of diffusion information which is also part of recent research works [34-37].

\section{Motion sensitivity}

Subject motion is known to affect the quality of the reconstructed transient-state image time-series, which then propagates to the estimation of tissue parameters [38, 39]. 3D QTI was found to be tolerant to marginal head movements so that we achieved image qualities of the parametric maps comparable to qualitative, state-of-the-art protocols. We attribute this to the fast acquisition based on undersampled spiral readouts, which repeatedly sample the $\mathrm{k}$-space center and are therefore more robust to motion already in the first place. This is particularly advantageous for severely diseased patients with difficulties to lie still during lengthy scanning sessions. However, initial experience also revealed that more pronounced patient motion can degrade the image quality of reconstructed image time-series and biases estimated parameter maps (Fig. 4). The axial, sagittal, and coronal views showcase that depending on the actual motion pattern, image quality is not homogeneously degraded in all spatial directions. For instance, despite the motion-caused image blurring, image quality in the sagittal direction of the motion-affected synthetic T2-weighted image is still comparable to the clinical T2-weighted PROPELLER acquisition with a native slice thickness of $3 \mathrm{~mm}$ and thus lower spatial resolution in this direction. Further, from the image artifacts that are apparent in the post-contrast T1-weighted MRI, it becomes clear that patient motion is also a major challenge in state-of-the-art conventional MRI. While patient motion manifests as diffuse image blurring in case of the spiral 3D QTI readout, we observe typical ghosting artifacts for the Cartesian readout scheme of the clinical postcontrast T1-weighted scan due to the unique frequencyand phase-encoding directions. Combining the 3D QTI 


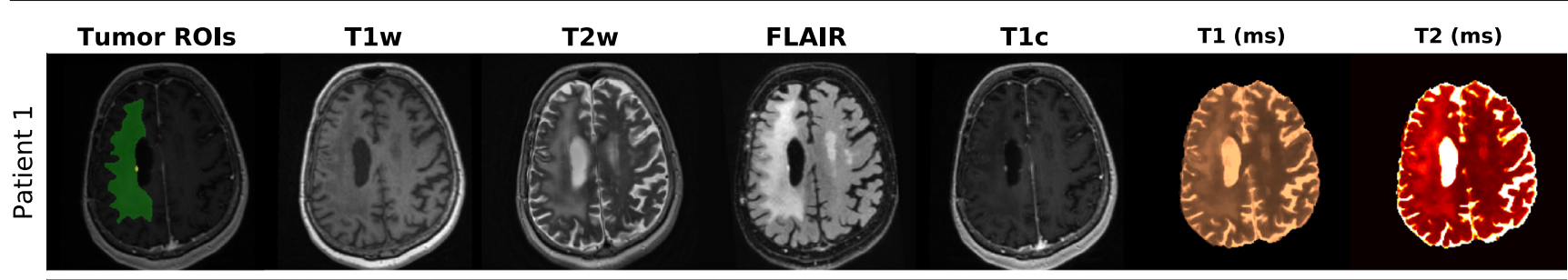
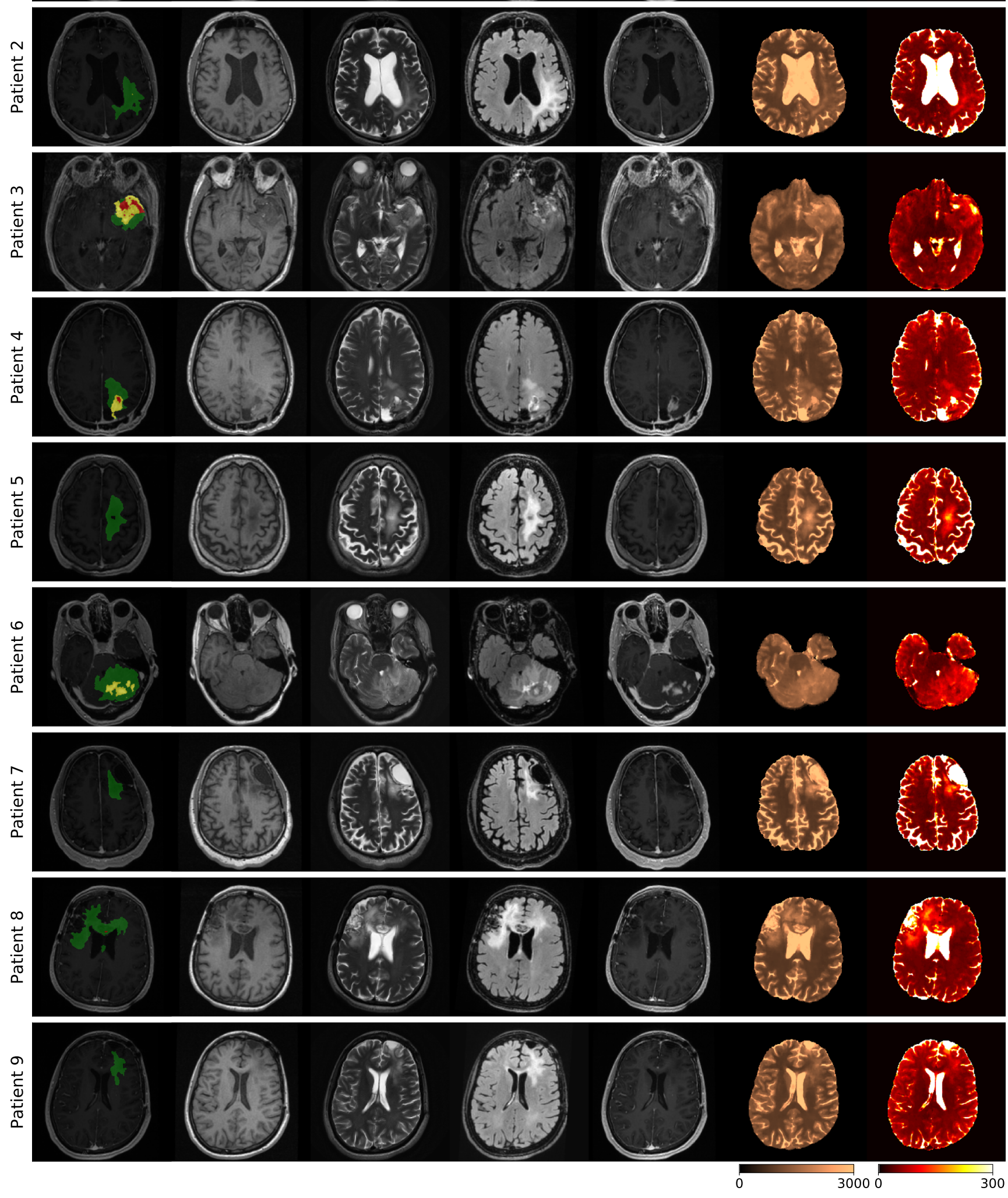
Fig. 6 Qualitative comparison of tumor patient cases. Expert ROIs (green: peritumoral edema, red: necrotic core/non-enhancing tumor, yellow: enhancing tumor) are shown together with clinical T1-weighted FSPGR, T2-weighted, FLAIR, Gd-enhanced T1-weighted FSPGR images and quantitative $\mathrm{T} 1$ and $\mathrm{T} 2$ maps

framework with a motion correction algorithm was previously shown to improve its robustness and can correct for patient motion [38]. Currently, this method can only correct for movements on a 7-s timescale, which could not sufficiently resolve the image degradation for the motionaffected patient case in our study.

In the same fashion as rigid head motion, the pulsating blood flow and the thereby induced pulsation of the cerebrospinal fluid (CSF) impact parameter estimation (Fig. 5). This is particularly seen in large vessels and in regions with high CSF pulsation, e.g., along the brainstem. Here, T2 values in the flowing blood are underestimated, which then reflects in lower signal intensities in the synthesized T2-weighted MRI compared to the clinical acquisition.

Given these findings, it is subject to our current and future work to also resolve motion on a faster scale, such as continuous rigid head motion, and to reduce sensitivity to physiological motion due to blood flow and/or brain pulsation.

\section{Application to quantitative characterization of tumor substructures}

\section{Current state-of-the-art}

Combination of advanced quantitative MR techniques together with contrast-weighted MRI has been shown to provide clinically relevant tissue information and is a key feature for precise tumor diagnostics: to date, stateof-the-art clinical routine MRI protocols, with acquisition times ranging from 20 to $60 \mathrm{~min}$, generally comprise preand post-contrast T1-weighted, T2-weighted, and FLAIR sequences, which can be extended by $\mathrm{T} 2 *$-weighted or susceptibility-weighted contrasts. Qualitative imaging is complemented by advanced quantitative MRI $[40,41]$ to capture tumor morphology and functionality, namely diffusion-weighted imaging and perfusion MRI [10]. Also, MR spectroscopy is used to improve brain tumor diagnostics and grading, although usually not as part of routine imaging. Diffusion tensor imaging and functional MRI provide essential information for surgery planning and guide tumor resection, as they inform the identification of tumor boundaries as well as localization of critical functional areas and neuron tracts.
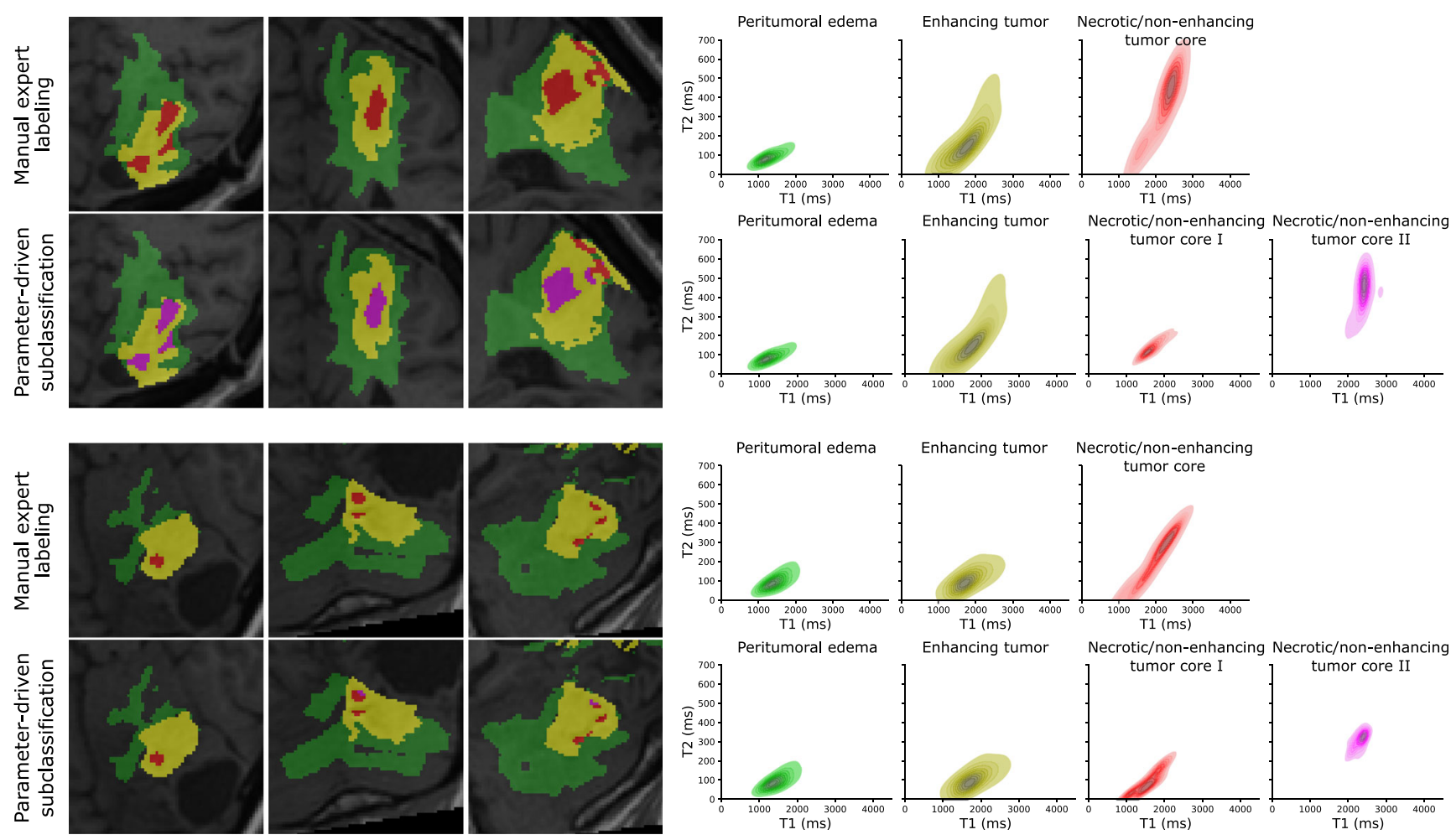

Enhancing tumor Necrotic/non-enhancing
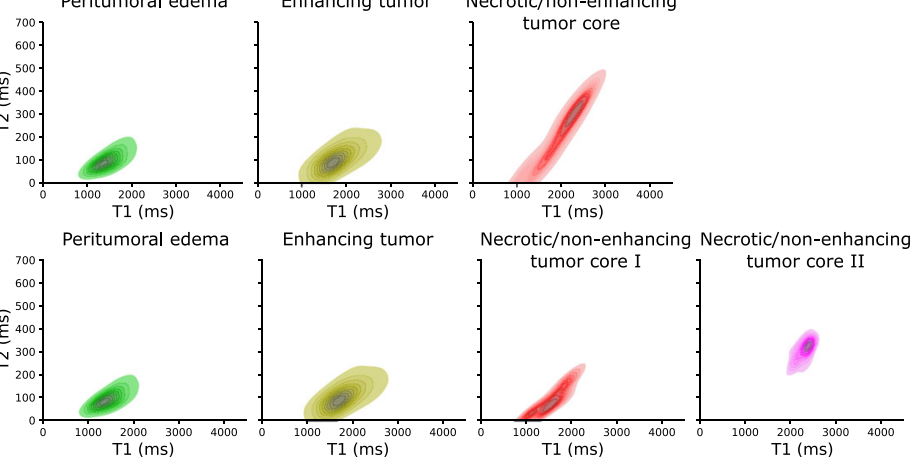

Fig. 7 Qualitative T1-T2-analysis based on manual ROI annotations together with additional explorative parameter-driven tumor subclassification for two representative patient cases. Classification of necrotic/nonenhancing tissue voxels based on quantitative $\mathrm{T} 1$ and $\mathrm{T} 2$ values can give more insights into the heterogenous structure, which we attribute to

fluidic (necrotic/non-enhancing tumor core II, magenta) and solid (necrotic/non-enhancing tumor core I, red) components, within the gross tumor regions (right). Expected spatial correlations of the two subcomponents are maintained as the back-projection of the T1-T2-based classification of necrotic and solid tissue results in connected annotations (left) 
a

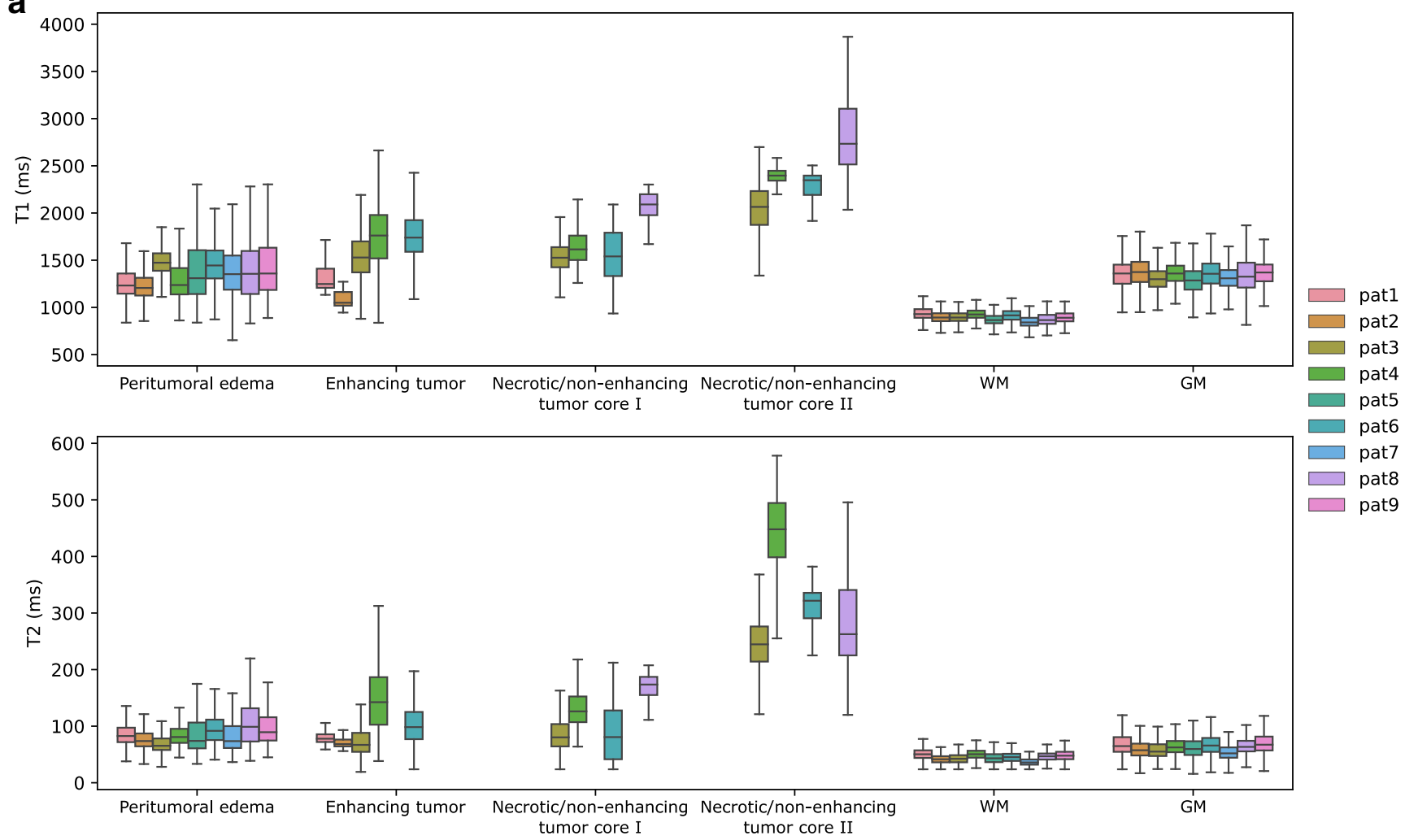

b

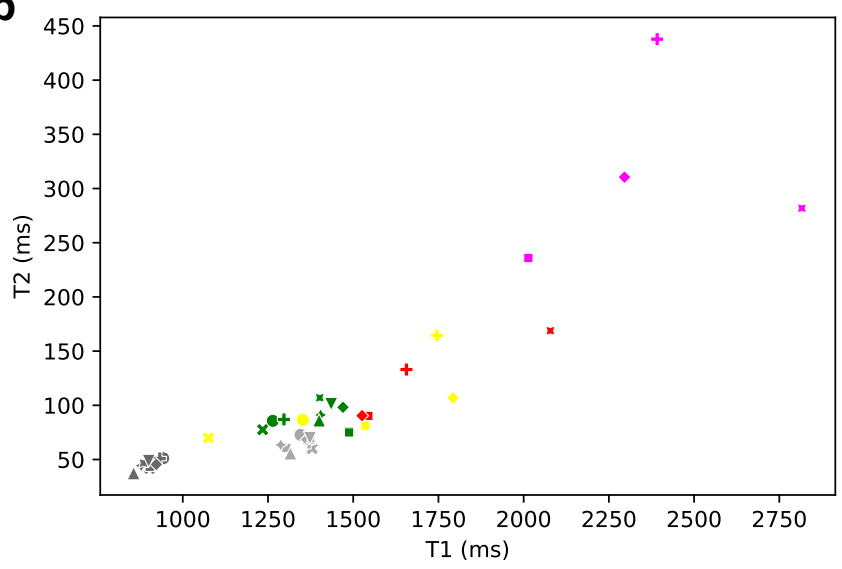

Fig. 8 Quantitative ROI-based parameter analysis. Boxplots of the patient-wise T1 and T2 parameter spaces (a) and a scatter plot of the respective mean $\mathrm{T} 1$ and $\mathrm{T} 2$ values $(\mathbf{b})$ indicate increased $\mathrm{T} 1$ and $\mathrm{T} 2$

Beyond the mentioned quantitative MRI schemes, which already made their way into clinical routine, several studies have shown that MR relaxometry can provide additional, clinically relevant information about critical tissue changes in gliomas that are not visible in contrastweighted MRIs [42-44]. Among other findings, quantitative T1 and T2 mapping has been demonstrated to aid earlier detection of tumor progression compared to standard contrast-weighted MR imaging, due to an increase in $\mathrm{T} 1$ and $\mathrm{T} 2$ values in recurring glioblastoma $[45,46]$. It has also been shown that detection of tissue changes in

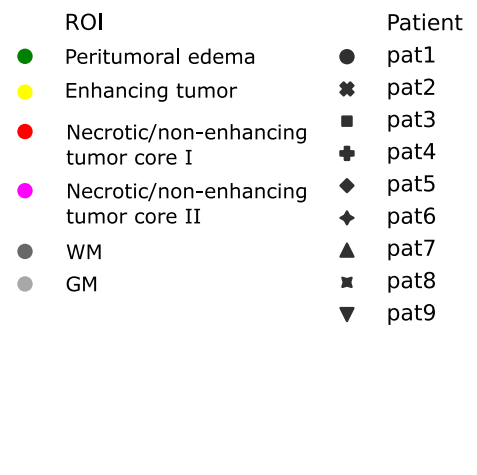

values in diseased, tumorous regions compared to healthy, contralateral WM and GM regions with high inter-subject consistency and small variance. Outliers in the boxplots are omitted for clarity

peritumoral regions can benefit from quantitative $\mathrm{T} 1$ and T2 mapping due to their earlier sensitivity compared to contrast-weighted MRI [47].

Although above mentioned methods have proven to offer critical measures for disease characterization and prognosis, they often require expensive off-line processing, involve casespecific tuning of sequence settings, or cannot meet the clinical time-constraints that challenge their standard use in clinical brain tumor imaging protocols.

A variety of advanced multiparametric relaxometry techniques $[12,13,48]$, including the pioneering work on MR 
Table 3 Quantitative summary of T1 and T2 values (mean, standard deviation) of the tumor ROIs and contralateral WM and GM regions

\begin{tabular}{lllllll}
\hline ROI & \multicolumn{2}{l}{ T1 (ms) } & & \multicolumn{2}{l}{ T2 (ms) } \\
\cline { 2 - 3 } & Mean & Std & & Mean & Std \\
\hline Enhancing tumor & 1685 & 340 & & 111 & 70 \\
Necrotic/non-enhancing tumor core I & 1550 & 195 & 92 & 38 \\
Necrotic/non-enhancing tumor core II & 2188 & 375 & 297 & 111 \\
Peritumoral edema & 1369 & 264 & 91 & 35 \\
WM & 903 & 76 & & 46 & 11 \\
GM & 1353 & 184 & 66 & 24 \\
\hline
\end{tabular}

fingerprinting (MRF) $[14,28]$, have been shown to offer fast, robust, and user-friendly quantitative MRI to be easily integrated into radiological practice. Its attractiveness in terms of scan times together with its high degree of repeatability [32, $49,50]$ makes these techniques attractive candidates for providing relaxometry-based biomarkers in day-to-day brain tumor diagnosis.

Despite the increasing number of recent works that focus on transient-state-based relaxometry techniques, there is only a modest number of studies to investigate their feasibility in disease-specific setups, such as glioma imaging [51-53]. These works have for example demonstrated the feasibility of MRF for brain tumor characterization. While these studies are based on 2D acquisitions with discrete slices placed in the tumor regions, our results in a patient cohort with a realistic, heterogenous clinical picture in terms of disease and treatment histories (Table 1, Fig. 6) demonstrate a fully 3D whole-brain quantitative analysis of tumor information captured in $\mathrm{T} 1$ and $\mathrm{T} 2$ estimates.

\section{Qualitative comparison of the tumor cases}

Our feasibility study of a variety of glioma patients showed that 3D QTI can be viable for tissue characterization and discrimination. Qualitatively, a ROI-based analysis of voxel-wise $\mathrm{T} 1$ and $\mathrm{T} 2$ relationships revealed homogenous distributions for peritumoral edema and enhancing tumor regions. In case of necrotic/nonenhancing tumor tissue, T1-T2 parameter spaces seemed to be composed of two classes, which we attributed to

Table 4 Quantitative summary of T1 and T2 values (mean, standard deviation) of WM and GM regions in a healthy volunteer

\begin{tabular}{llllll}
\hline & \multicolumn{2}{l}{ T1 (ms) } & & \multicolumn{2}{l}{ T2 (ms) } \\
\cline { 2 - 3 } \cline { 5 - 6 } ROI & Mean & Std & & Mean & Std \\
\hline GM & 1331 & 137 & 66 & 12 \\
WM & 871 & 77 & 45 & 7 \\
\hline
\end{tabular}

fluidic and solid tissue components within the gross tumor. Building on the clinical expert annotation, we aimed to gain more insight into necrotic/non-enhancing tumor tissue and explored whether a Gaussian mixture model can disentangle these two voxel classes (Fig. 7). Linking the thereby identified tissue subclasses to the anatomical space reveal spatially well-connected annotations. In clinical routine tumor annotation, solid and fluidic parts within the necrotic/non-enhancing tumor regions are generally not differentiated and comprised in one overall ROI. That is why we do not have a reference annotation to compare the results of the Gaussian mixture model with. Nevertheless, we believe that our explorative analysis is an illustrative example of how quantitative, multiparametric measures of the underlying relaxation times can complement tumor annotations that are generally based on qualitative, visual abnormalities in contrastweighted MR image data.

\section{Quantitative analysis of tumor substructures}

Quantitative analysis (Fig. 8 and Table 3) substantiated and complemented these qualitative findings. We observed that tumor tissue, i.e., enhancing tumor, necrotic/non-enhancing tumor core I + II, as well as peritumoral edema, exhibit higher $\mathrm{T} 1$ and $\mathrm{T} 2$ values than healthy contralateral $\mathrm{WM}$, as qualitatively suggested from Fig. 6. These findings are in line with initial clinical outcomes of MR fingerprinting [51], supporting the potential of 3D QTI as an image-based biomarker for glioma diagnosis.

Figure 8 also suggests well-defined parameter spaces for healthy WM and GM regions among the patient cohort with only small inter-subject variations. Also, T1 and T2 mapping in non-diseased tissue was found to agree well with our results for healthy volunteer data, as suggested by Table 3 and Table 4, and is consistent with previously reported values $[18,38,54,55]$. Furthermore, mean $\mathrm{T} 1$ and $\mathrm{T} 2$ values of the two gross tumor subclasses are well distinguished. That is, mean $\mathrm{T} 1$ and $\mathrm{T} 2$ values for necrotic/non-enhancing tumor core II, which we attributed to the fluidic subcomponent, are constantly higher than for necrotic tumor/non-enhancing core I. This finding agrees with the fact that $\mathrm{T} 1$ and $\mathrm{T} 2$ relaxation times are sensitive to tissue composition and microstructure $[56,57]$.

Overall, T1 and T2 values suggest a clear distinction between peritumoral edema and contralateral WM (Fig. 8b). Also, mean T2 values in peritumoral edema were higher than for contralateral GM in all patients. Mean T1 values in peritumoral edema were lower compared to necrotic/nonenhancing tumor regions. For mean T2 values, this is only observed in case of patients 4 and 8. As observed from Fig. $8 \mathrm{~b}$, enhancing tumor overlays with peritumoral edema and necrotic/non-enhancing tumor core I in the T1-T2 space. 
Enhancing tumor is segmented as those regions within the gross tumor that exhibit positive Gd enhancement in the Gdenhanced T1-weighted MRI. Native pre-contrast T1 maps hence do not necessarily capture this differentiation.

Due to the observed inter-subject consistency and stability of T1 and T2 values of healthy and diseased tissue, which is in line with previously performed repeatability studies $[32,58$, 59], we believe that not only diagnosis but also treatment planning and monitoring as well as prognostic, longitudinal assessment might benefit from an integration of 3D QTI into standard clinical imaging.

While conventional contrast-weighted MRI can also capture tissue heterogeneity in tumor texture, it always represents weighted information of a combination of relaxation and tissue parameters. Also, qualitative MRI information is known to depend on the actual scanner settings, which can vary from day to day due to different pre-scan conditions or gain tunings, hampering longitudinal or inter-subject comparisons.

Despite the mentioned benefits of fast multiparametric mapping techniques, we emphasize that both quantitative and qualitative MRI are generally limited to macroscopic voxel sizes. Resulting partial volume effects, e.g., at the border of distinct tissue types, are known to mask the underlying cellular tissue characteristics, only providing an effective voxel value of the respective biomarker. However, aiming for high isotropic resolution with sufficient SNR, as in case of 3D QTI, minimizes partial volume effects in the first place. Comparing the synthetic T2-weighted MRI generated from the 3D QTI scan with the clinical 2D reference with higher slice thickness in Fig. 3 illustrates this resolution benefit, e.g., for tumor delineation.

Nevertheless, with 3D QTI producing reliable, reproducible, quantitative image data, it may be a valuable tool for the harmonization of imaging data with the opportunity to make multi-timepoint, multi-subject, multi-vendor, and multi-center studies easier. As such, it can offer a rich set of standardized, comprehensive image data with the potential to also advance methodological developments along the large spectrum of AI-based decision support, i.e., segmentation algorithms or tumor growth modeling.

\section{Limitations}

A clear limitation to this study is the rather small sample size. We aimed to present an initial clinical case study demonstrating the feasibility of 3D QTI for integration into routine glioma imaging protocols. As such, the rather small but heterogenous study cohort, comprising both pre- and post-surgery treatment stages as well as different tumor grades, allowed us to nevertheless cover the bandwidth of patient cases in clinical radiological practice.

\section{Outlook}

Given our initial clinical results, we would like to employ 3D QTI also for longitudinal follow-up of glioma patients to evaluate its potential for monitoring and quantifying treatment response in individual subjects to support individualized therapy decisions with the aim of personalized medicine. We also plan to investigate how deep learning-based segmentation and interpretation of images $[60,61]$ could benefit from complementing or even replacing the to-date mainly qualitative data bases with quantitative imaging biomarkers, e.g., as provided by 3D QTI. Furthermore, we believe that extending the method to jointly encode relaxation and diffusion parameters as proposed in [37] with fully 3D, high isotropic resolution, and whole-brain coverage can further improve its attractiveness for integration into clinical glioma imaging and is hence subject of our current work. We also aim to further increase motion robustness of the transient-state encoding scheme. Building on the previously presented retrospective motion correction [38], we are optimistic that we can further develop this approach to account and correct for head motion on a faster time scale. We might also benefit from recent advances on prospective motion correction [62]. To reduce the sensitivity to nonrigid motion, such as blood flow and/or brain pulsation, in first place, it is subject to our future work to investigate potential refinements in the sequence design. The experience with $3 \mathrm{D}$ QTI in neuro applications also motivates us to target potential diagnostic scenarios outside the brain as well as in combination with contrast enhancement $[63,64]$.

\section{Conclusion}

3D QTI demonstrated to reliably identify tissue and hence tumor heterogeneity that is captured in $\mathrm{T} 1$ and $\mathrm{T} 2$ relaxation times under tight clinical time constraints. As such, it offers comprehensive tissue assessment of tumor substructures with the potential to improve disease characterization in brain tumor patients. This is essential to find the optimal treatment strategy and to monitor treatment response along the course of disease.

\section{Appendix}



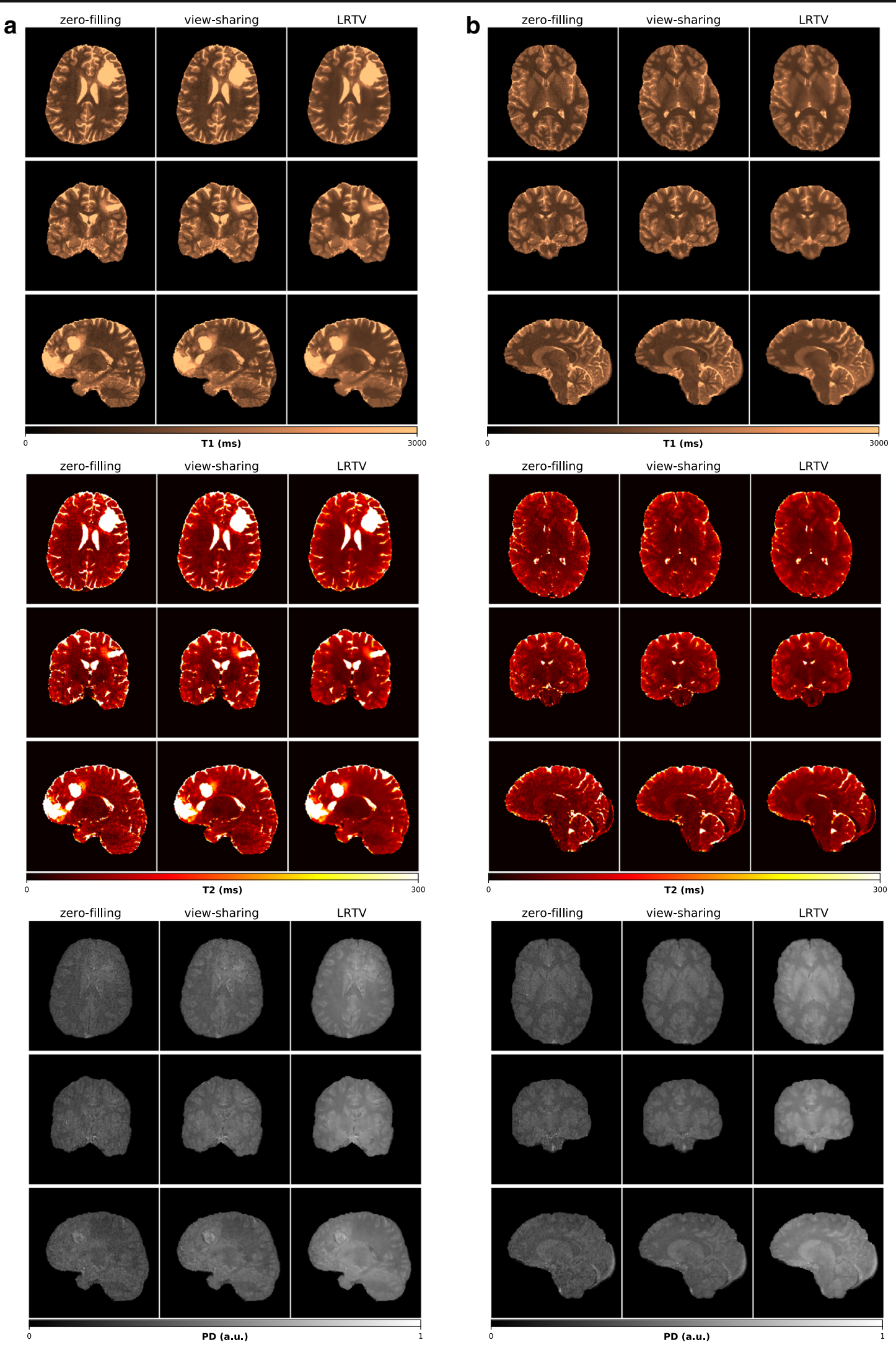

Fig. 9 Parametric maps of T1, T2, and PD obtained with zero-filling, kspace weighted view-sharing, and LRTV reconstructions and subsequent dictionary matching for a representative patient case (a) and a healthy volunteer (b). Parameter estimation is consistent across all reconstruction

approaches. View-sharing reconstruction provides better image quality in the quantitative maps than zero-filling with best reduction of undersampling artifacts obtained with LRTV reconstruction 
a

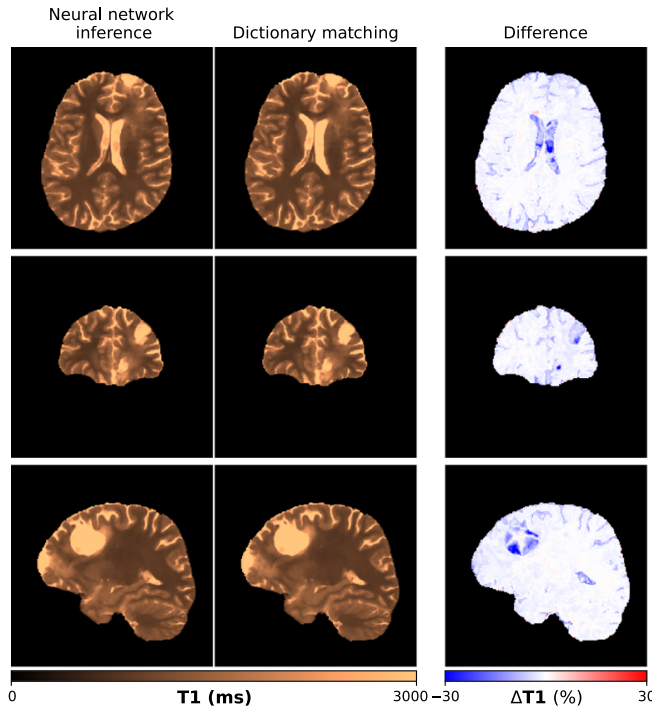

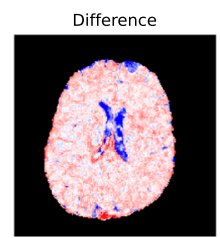
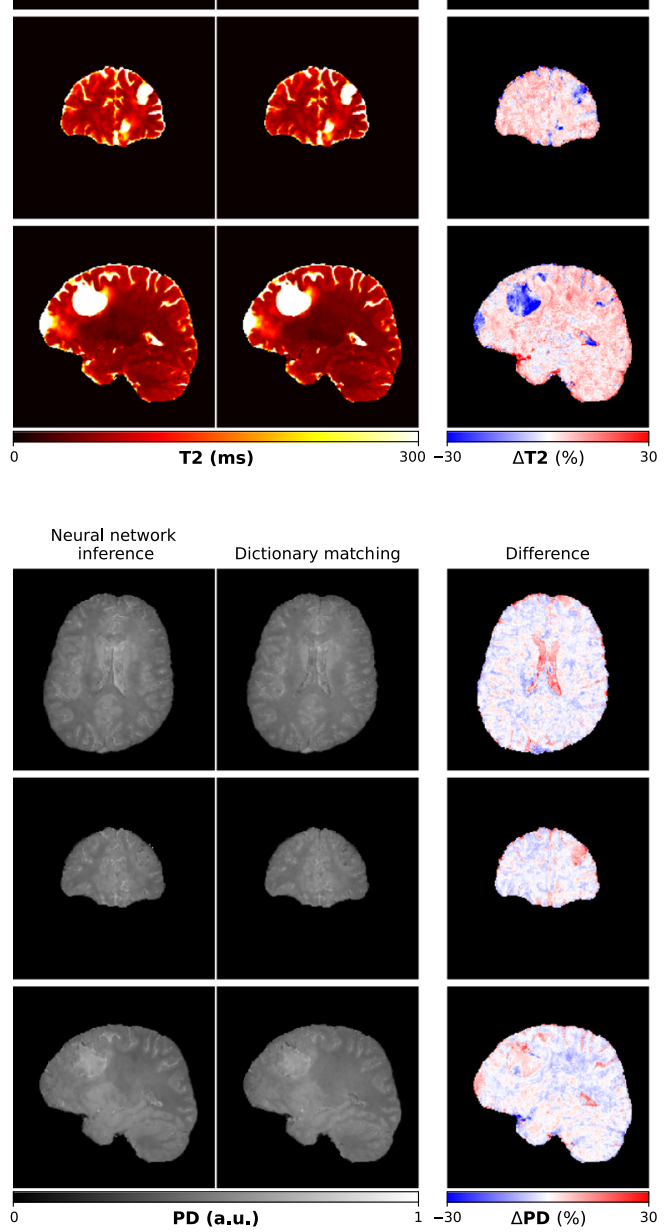

b
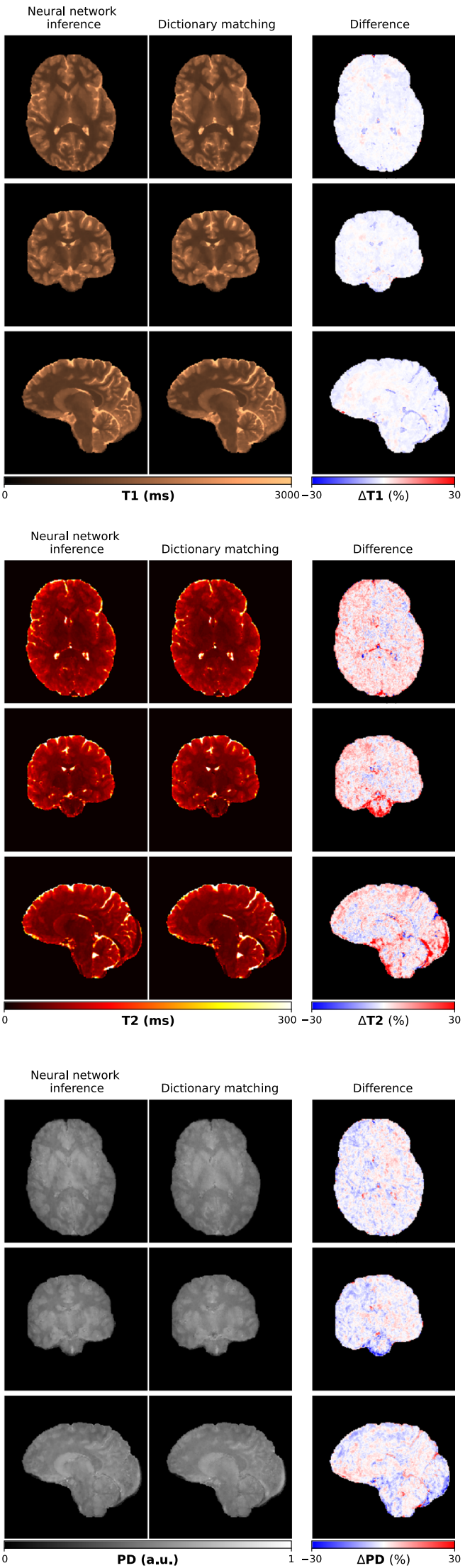
Fig. 11 Contrast-weighted image synthesis for a representative patient case. Sagittal and coronal views in correspondence to the axial views in Fig. 3 are shown
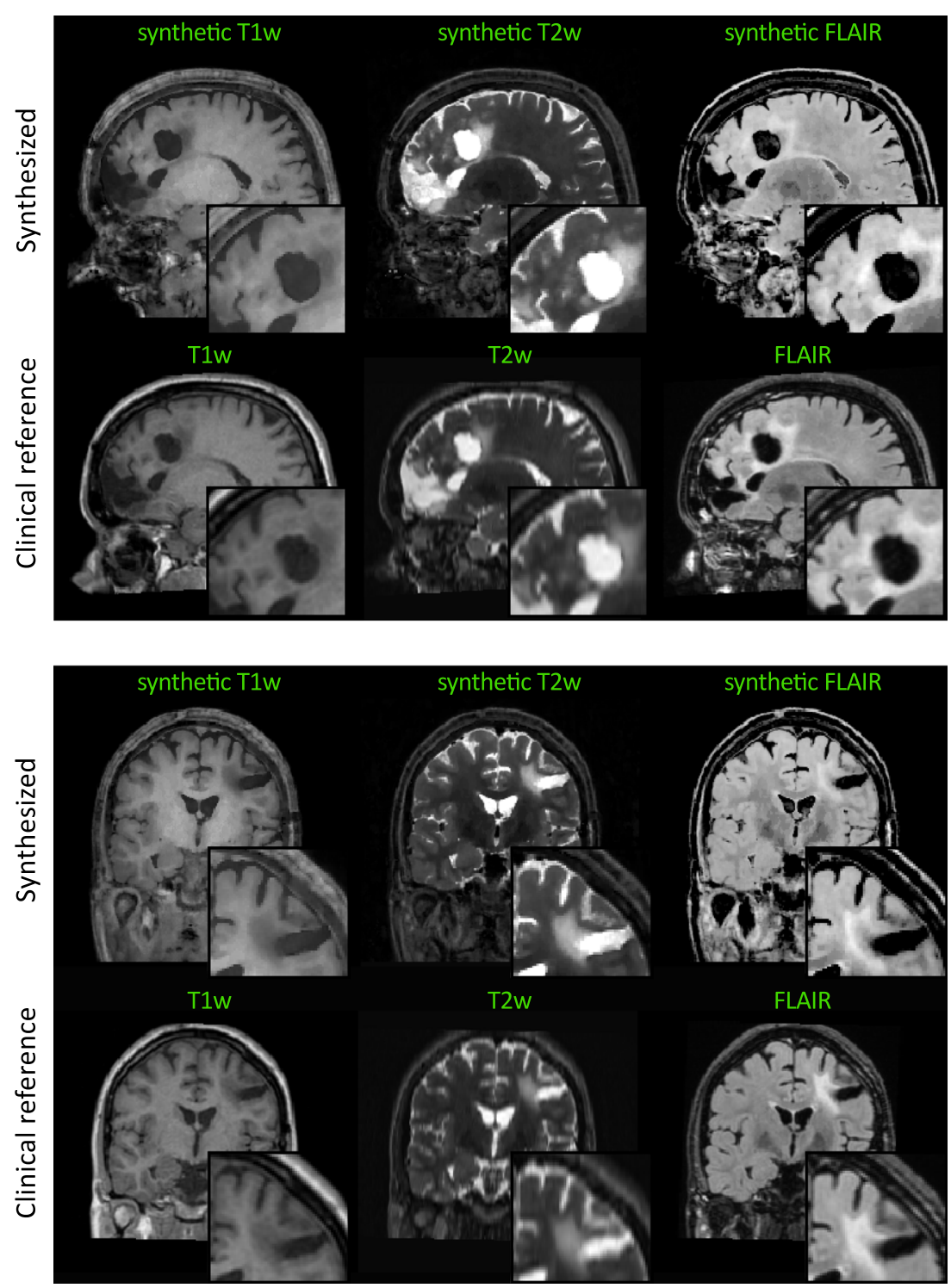

4 Fig. 10 Neural network-based parameter estimation for a representative patient case (top) and a healthy volunteer (bottom). T1, T2, and PD maps obtained from voxel-wise neural network inference are consistent with dictionary matching results. The parameter maps are computed from SVD-compressed image-series after LRTV reconstruction 
Fig. 12 Sensitivity to rigid head motion. Sagittal and coronal views in correspondence to the axial views in Fig. 4 are shown
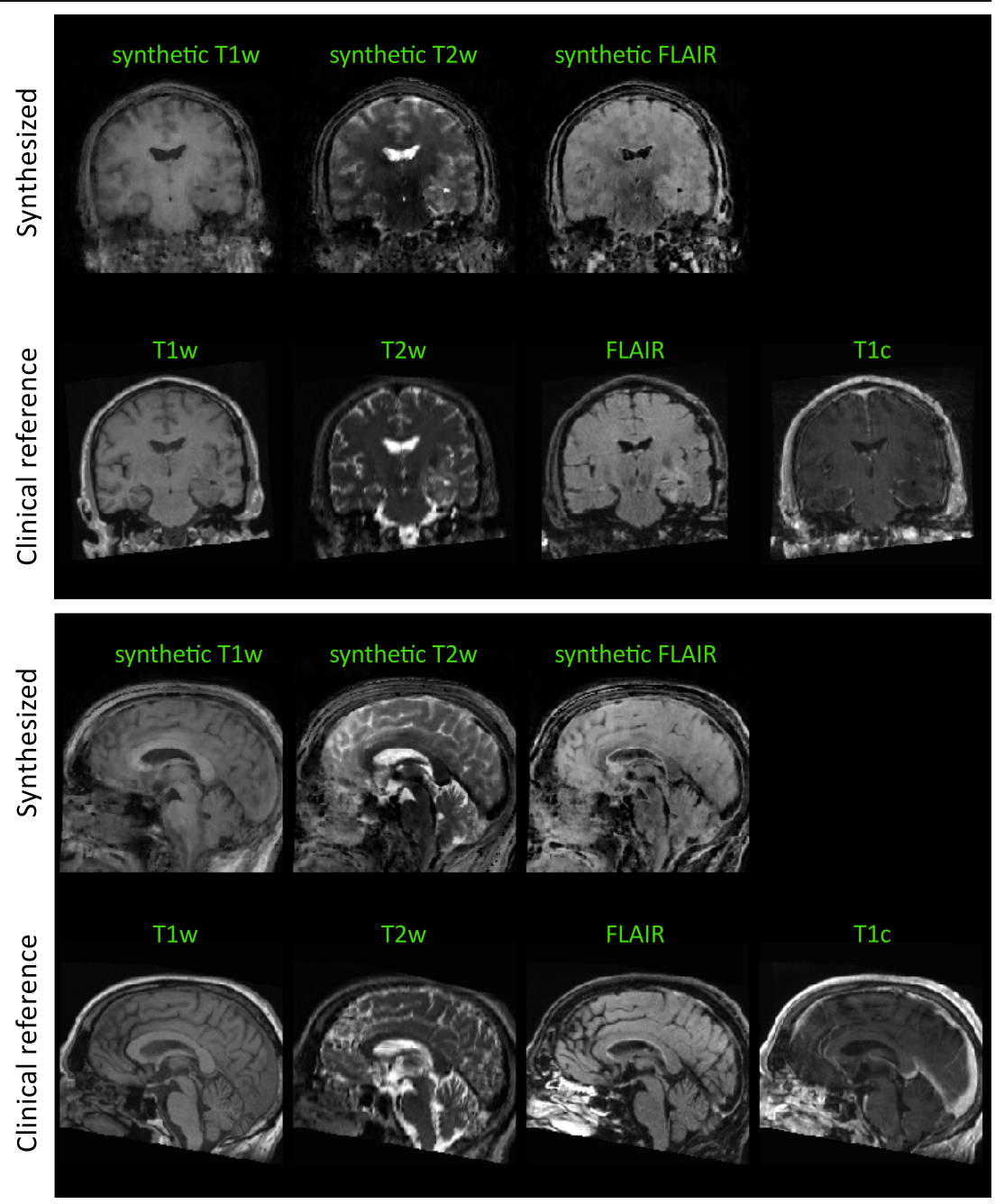

Author contribution Conceptualization: C.M.P, M.S., B.H.M, M.I.M., J.A.H.-T.; methodology: C.M.P, M.G., P.A.G., G.B., M.C., R.F.S.; data collection: L.N.-G., M.S., J.A.H.-T.; data analysis: C.M.P, L.N.-G., F.K., S.E., L.G., B.W.; writing-original draft preparation: C.M.P.; writingreview and editing: all authors; supervision: B.H.M., M.I.M., J.A.H.-T.; all authors read and approved the final manuscript.

Funding Open Access funding enabled and organized by Projekt DEAL. C.M.P., S.E., B.H.M., and M.I.M. are supported by Deutsche Forschungsgemeinschaft (DFG) through Research Training Group GRK 2274 and TUM International Graduate School of Science and Engineering (IGSSE), GSC 81. C.M.P., S.E., M.I.M. receive funding from the European Union's Horizon 2020 research and innovation programme under grant agreement No. 952172. Patient data were acquired under the GE Healthcare work statement B-GEHC-05.

Data availability The data presented in this study are available on reasonable request from the corresponding author. The data are not publicly available due to restrictions on the use of confidential data in the written consent provided by participants.

\section{Declarations}

Ethics approval This study was in accordance with the ethical standards of the institutional and/or national research committee and with the 1964 Helsinki declaration and its later amendments or comparable ethical standards. Approval was granted by the local ethics board.

Consent to participate All participants included in this study provided written informed consent.

Consent for publication All participants included in this study signed informed consent regarding publishing their MRI data.

Conflict of Interest C.M.P, R.F.S., and M.I.M are employees at GE Healthcare, Munich, Germany. M.S. received an honorarium (paid to institution) from Parexel Ltd. for EORTC-1410 and speaker fees (paid to institution) from GE Healthcare. All other authors declare no competing interests. 
Open Access This article is licensed under a Creative Commons Attribution 4.0 International License, which permits use, sharing, adaptation, distribution and reproduction in any medium or format, as long as you give appropriate credit to the original author(s) and the source, provide a link to the Creative Commons licence, and indicate if changes were made. The images or other third party material in this article are included in the article's Creative Commons licence, unless indicated otherwise in a credit line to the material. If material is not included in the article's Creative Commons licence and your intended use is not permitted by statutory regulation or exceeds the permitted use, you will need to obtain permission directly from the copyright holder. To view a copy of this licence, visit http://creativecommons.org/licenses/by/4.0/.

\section{References}

1. Perry A, Wesseling P (2016) Histologic classification of gliomas. Handb Clin Neurol 134:71-95. https://doi.org/10.1016/B978-0-12802997-8.00005-0

2. Holland EC (2001) Progenitor cells and glioma formation. Curr Opin Neurol 14:683-688. https://doi.org/10.1097/00019052200112000-00002

3. Thust SC, Heiland S, Falini A, Jäger HR, Waldman AD, Sundgren PC, Godi C, Katsaros VK, Ramos A, Bargallo N, Vernooij MW, Yousry T, Bendszus M, Smits M (2018) Glioma imaging in Europe: a survey of 220 centres and recommendations for best clinical practice. Eur Radiol 28:3306-3317. https://doi.org/10. 1007/s00330-018-5314-5

4. Louis DN, Ohgaki H, Wiestler OD, Cavenee WK (2016) WHO Classification of Tumours of the Central Nervous System, 4th edn. International Agency for Research on Cancer, France

5. van den Bent MJ, Smits M, Kros JM, Chang SM (2017) Diffuse infiltrating oligodendroglioma and astrocytoma. J Clin Oncol 35: 2394-2401. https://doi.org/10.1200/JCO.2017.72.6737

6. Fouke SJ, Benzinger T, Gibson D, Ryken TC, Kalkanis SN, Olson JJ (2015) The role of imaging in the management of adults with diffuse low grade glioma: a systematic review and evidence-based clinical practice guideline. J Neuro-Oncol 125:457-479. https://doi. org/10.1007/s11060-015-1908-9

7. Olsen KI, Schroeder P, Corby R, Vucic I, Bardo DME (2005) Advanced magnetic resonance imaging techniques to evaluate CNS glioma. Expert Rev Neurother 5:3-11. https://doi.org/10. 1586/14737175.5.6.S3

8. Kao H-W, Chiang S-W, Chung H-W, Tsai FY, Chen CY (2013) Advanced MR imaging of gliomas: an update. Biomed Res Int 2013:2013-2014. https://doi.org/10.1155/2013/970586

9. Wank M, Schilling D, Schmid TE, Meyer B, Gempt J, Barz M, Schlegel J, Liesche F, Kessel K, Wiestler B, Bette S, Zimmer C, Combs S (2018) Human glioma migration and infiltration properties as a target for personalized radiation medicine. Cancers (Basel) 10:10. https://doi.org/10.3390/cancers 10110456

10. Kalpathy-Cramer J, Gerstner ER, Emblem KE, Andronesi OC, Rosen B (2014) Advanced magnetic resonance imaging of the physical processes in human glioblastoma. Cancer Res 74:46224637. https://doi.org/10.1158/0008-5472.CAN-14-0383

11. Ellingson BM, Bendszus M, Boxerman J et al (2015) Consensus recommendations for a standardized Brain Tumor Imaging Protocol in clinical trials. Neuro-Oncology 17:1188-1198. https://doi.org/ 10.1093/neuonc/nov095

12. Deoni SCL, Rutt BK, Peters TM (2003) Rapid combined T1 and T2 mapping using gradient recalled acquisition in the steady state. Magn Reson Med 49:515-526. https://doi.org/10.1002/mrm.10407

13. Warntjes JBM, Leinhard OD, West J, Lundberg P (2008) Rapid magnetic resonance quantification on the brain: optimization for clinical usage. Magn Reson Med 60:320-329. https://doi.org/10. $1002 / \mathrm{mrm} .21635$

14. Ma D, Gulani V, Seiberlich N, et. al. (2013) Magnetic resonance fingerprinting. Nature 495:187-192. https://doi.org/10.1038/ nature11971

15. Sbrizzi A, van der Heide O, Cloos M et al (2018) Fast quantitative MRI as a nonlinear tomography problem. Magn Reson Imaging 46: 56-63. https://doi.org/10.1016/j.mri.2017.10.015

16. Gómez PA, Molina-Romero M, Buonincontri G, Menzel MI, Menze BH (2019) Designing contrasts for rapid, simultaneous parameter quantification and flow visualization with quantitative transient-state imaging. Sci Rep 9:8468. https://doi.org/10.1038/ s41598-019-44832-w

17. van der Heide O, Sbrizzi A, van den Berg CAT (2020) Accelerated MR-STAT reconstructions using sparse Hessian approximations. IEEE Trans Med Imaging 39:3737-3748. https://doi.org/10.1109/ TMI.2020.3003893

18. Gómez PA, Cencini M, Golbabaee M, Schulte RF, Pirkl C, Horvath I, Fallo G, Peretti L, Tosetti M, Menze BH, Buonincontri G (2020) Rapid three-dimensional multiparametric MRI with quantitative transient-state imaging. Sci Rep 10:13769. https://doi.org/10. 1038/s41598-020-70789-2

19. da Cruz LCH, Rodriguez I, Domingues RC et al (2011) Pseudoprogression and pseudoresponse: imaging challenges in the assessment of posttreatment glioma. Am J Neuroradiol 32: 1978-1985. https://doi.org/10.3174/ajnr.A2397

20. Hargreaves BA, Vasanawala SS, Pauly JM, Nishimura DG (2001) Characterization and reduction of the transient response in steadystate MR imaging. Magn Reson Med 46:149-158. https://doi.org/ $10.1002 / \mathrm{mrm} .1170$

21. Golbabaee M, Buonincontri G, Pirkl CM, Menzel MI, Menze BH, Davies M, Gómez PA (2021) Compressive MRI quantification using convex spatiotemporal priors and deep encoder-decoder networks. Med Image Anal 69:101945. https://doi.org/10.1016/j. media.2020.101945

22. Cruz G, Schneider T, Bruijnen T, Gaspar AS, Botnar RM, Prieto C (2018) Accelerated magnetic resonance fingerprinting using softweighted key-hole (MRF-SOHO). PLoS One 13:e0201808. https:// doi.org/10.1371/journal.pone.0201808

23. Knoll F, Schwarzl A, Diwoky C, Sodickson DK (2014) gpuNUFFT - An Open-Source GPU Library for 3D Gridding with Direct Matlab Interface. In: Proceedings of the 22th Annual Meeting of International Society for Magnetic Resonance in Medicine (ISMRM). Milan, Italy

24. Walsh DO, Gmitro AF, Marcellin MW (2000) Adaptive reconstruction of phased array MR imagery. Magn Reson Med 43: 682-690. https://doi.org/10.1002/(sici)1522-2594(200005)43: $5<682:$ :aid-mrm10>3.0.co;2-g

25. Hoppe E, Körzdörfer G, Würfl T, Wetzl J, Lugauer F, Pfeuffer J, Maier A (2017) Deep learning for magnetic resonance fingerprinting: a new approach for predicting quantitative parameter values from time series. Stud Health Technol Inform 243:202-206

26. Cohen O, Zhu B, Rosen MS (2018) MR fingerprinting Deep RecOnstruction NEtwork (DRONE). Magn Reson Med 80:885894. https://doi.org/10.1002/mrm.27198

27. Weigel M (2015) Extended phase graphs: dephasing, RF pulses, and echoes - pure and simple. J Magn Reson Imaging 41:266-295. https://doi.org/10.1002/jmri.24619

28. Jiang Y, Ma D, Seiberlich N, Gulani V, Griswold MA (2015) MR fingerprinting using fast imaging with steady state precession (FISP) with spiral readout. Magn Reson Med 74:1621-1631. https://doi.org/10.1002/mrm.25559

29. Yushkevich PA, Piven J, Hazlett HC, Smith RG, Ho S, Gee JC, Gerig G (2006) User-guided 3D active contour segmentation of anatomical structures: significantly improved efficiency and 
reliability. Neuroimage 31:1116-1128. https://doi.org/10.1016/j. neuroimage.2006.01.015

30. Avants BB, Tustison NJ, Song G, Cook PA, Klein A, Gee JC (2011) A reproducible evaluation of ANTs similarity metric performance in brain image registration. Neuroimage 54:2033-2044. https://doi.org/10.1016/j.neuroimage.2010.09.025

31. Zhang Y, Brady M, Smith S (2001) Segmentation of brain MR images through a hidden Markov random field model and the expectation-maximization algorithm. IEEE Trans Med Imaging 20:45-57. https://doi.org/10.1109/42.906424

32. Buonincontri G, Kurzawski JW, Kaggie JD, Matys T, Gallagher FA, Cencini M, Donatelli G, Cecchi P, Cosottini M, Martini N, Frijia F, Montanaro D, Gómez PA, Schulte RF, Retico A, Tosetti M (2021) Three dimensional MRF obtains highly repeatable and reproducible multi-parametric estimations in the healthy human brain at $1.5 \mathrm{~T}$ and $3 \mathrm{~T}$. NeuroImage 226:117573. https://doi.org/10. 1016/j.neuroimage.2020.117573

33. Hattingen E, Müller A, Jurcoane A et al (2017) Value of quantitative magnetic resonance imaging T1-relaxometry in predicting contrast-enhancement in glioblastoma patients. Oncotarget 8: 53542-53551. https://doi.org/10.18632/oncotarget.18612

34. Jiang Y, Hamilton JI, Wright KL, et al (2016) Simultaneous quantification of $\mathrm{T} 1, \mathrm{~T} 2$ and diffusion with diffusion-weighted driveequilibrium prepared magnetic resonance fingerprinting. In: Proceedings of the 24th Annual Meeting of International Society for Magnetic Resonance in Medicine (ISMRM). Singapore

35. Jiang Y, Hamilton JI, Lo W-C, et al (2017) Simultaneous T1, T2 and diffusion quantification using multiple contrast prepared magnetic resonance fingerprinting. In: Proceedings of the 25th Annual Meeting of International Society for Magnetic Resonance in Medicine (ISMRM). Honolulu, HI, USA

36. Rieger B, Akçakaya M, Schad L, Weingärtner S (2018) Simultaneous quantification of T1, T2 and apparent diffusion coefficient using magnetic resonance fingerprinting based on echo planar imaging. In: Proceedings of the 26th Annual Meeting of International Society for Magnetic Resonance in Medicine (ISMRM). Paris, France

37. Pirkl CM, Gomez PA, Lipp I, et al (2020) Deep learning-based parameter mapping for joint relaxation and diffusion tensor MR fingerprinting. In: Third Conference on Medical Imaging with Deep Learning. PMLR, pp 638-654

38. Kurzawski JW, Cencini M, Peretti L, Gómez PA, Schulte RF, Donatelli G, Cosottini M, Cecchi P, Costagli M, Retico A, Tosetti M, Buonincontri G (2020) Retrospective rigid motion correction of three-dimensional magnetic resonance fingerprinting of the human brain. Magn Reson Med 84:2606-2615. https://doi.org/10.1002/ mrm. 28301

39. Lu L, Chen Y, Shen C, Lian J, Das S, Marks L, Lin W, Zhu T (2020) Initial assessment of 3D magnetic resonance fingerprinting (MRF) towards quantitative brain imaging for radiation therapy. Med Phys 47:1199-1214. https://doi.org/10.1002/mp.13967

40. Seow P, Wong JHD, Ahmad-Annuar A, Mahajan A, Abdullah NA, Ramli N (2018) Quantitative magnetic resonance imaging and radiogenomic biomarkers for glioma characterisation: a systematic review. Br J Radiol 91:20170930. https://doi.org/10.1259/bjr. 20170930

41. Guzmán-De-Villoria JA, Mateos-Pérez JM, Fernández-García P et al (2014) Added value of advanced over conventional magnetic resonance imaging in grading gliomas and other primary brain tumors. Cancer Imaging 14:35. https://doi.org/10.1186/s40644-0140035-8

42. Komiyama M, Yagura H, Baba M, Yasui T, Hakuba A, Nishimura S, Inoue Y (1987) MR imaging: possibility of tissue characterization of brain tumors using T1 and T2 values. AJNR Am J Neuroradiol 8:65-70
43. Just M, Thelen M (1988) Tissue characterization with T1, T2, and proton density values: results in 160 patients with brain tumors. Radiology 169:779-785. https://doi.org/10.1148/radiology.169.3. 3187000

44. Newman S, Haughton VM, Yetkin Z, Breger R, Czervionke LF, Williams AL, Ho KC, Papke RA, Rimm AA, Fischer ME, Meyer GA, Asleson R (1993) T1, T2 and proton density measurements in the grading of cerebral gliomas. Eur Radiol 3:49-52. https://doi. org/10.1007/BF00173524

45. Lescher S, Jurcoane A, Veit A, Bähr O, Deichmann R, Hattingen E (2015) Quantitative T1 and T2 mapping in recurrent glioblastomas under bevacizumab: earlier detection of tumor progression compared to conventional MRI. Neuroradiology 57:11-20. https://doi. org/10.1007/s00234-014-1445-9

46. Hattingen E, Jurcoane A, Daneshvar K, Pilatus U, Mittelbronn M, Steinbach JP, Bahr O (2013) Quantitative T2 mapping of recurrent glioblastoma under bevacizumab improves monitoring for nonenhancing tumor progression and predicts overall survival. NeuroOncology 15:1395-1404. https://doi.org/10.1093/neuonc/not105

47. Blystad I, Warntjes JBM, Smedby Ö, Lundberg P, Larsson EM, Tisell A (2017) Quantitative MRI for analysis of peritumoral edema in malignant gliomas. PLoS One 12:e0177135. https://doi.org/10. 1371/journal.pone. 0177135

48. Wang F, Dong Z, Reese TG, Bilgic B, Katherine Manhard M, Chen J, Polimeni JR, Wald LL, Setsompop K (2019) Echo planar timeresolved imaging (EPTI). Magn Reson Med 81:3599-3615. https:// doi.org/10.1002/mrm.27673

49. Jiang Y, Ma D, Keenan KE, Stupic KF, Gulani V, Griswold MA (2017) Repeatability of magnetic resonance fingerprinting T1 and T2 estimates assessed using the ISMRM/NIST MRI system phantom. Magn Reson Med 78:1452-1457. https://doi.org/10.1002/ mrm.26509

50. Panda A, Chen Y, Ropella-Panagis K, Ghodasara S, Stopchinski M, Seyfried N, Wright K, Seiberlich N, Griswold M, Gulani V (2019) Repeatability and reproducibility of 3D MR fingerprinting relaxometry measurements in normal breast tissue. J Magn Reson Imaging 50:1133-1143. https://doi.org/10.1002/jmri.26717

51. Badve C, Yu A, Dastmalchian S, Rogers M, Ma D, Jiang Y, Margevicius S, Pahwa S, Lu Z, Schluchter M, Sunshine J, Griswold M, Sloan A, Gulani V (2017) Magnetic resonance fingerprinting of adult brain tumors: initial experience. AJNR Am J Neuroradiol 38:492-499. https://doi.org/10.3174/ajnr.A5035

52. Dastmalchian S, Kilinc O, Onyewadume L, Tippareddy C, McGivney D, Ma D, Griswold M, Sunshine J, Gulani V, Barnholtz-Sloan JS, Sloan AE, Badve C (2020) Radiomic analysis of magnetic resonance fingerprinting in adult brain tumors. Eur $\mathrm{J}$ Nucl Med Mol Imaging. https://doi.org/10.1007/s00259-02005037-w

53. Haubold J, Demircioglu A, Gratz M, Glas M, Wrede K, Sure U, Antoch G, Keyvani K, Nittka M, Kannengiesser S, Gulani V, Griswold M, Herrmann K, Forsting M, Nensa F, Umutlu L (2020) Non-invasive tumor decoding and phenotyping of cerebral gliomas utilizing multiparametric 18F-FET PET-MRI and MR Fingerprinting. Eur J Nucl Med Mol Imaging 47:1435-1445. https://doi.org/10.1007/s00259-019-04602-2

54. Ma D, Jiang Y, Chen Y, McGivney D, Mehta B, Gulani V, Griswold M (2018) Fast 3D magnetic resonance fingerprinting for a whole-brain coverage. Magn Reson Med 79:2190-2197. https://doi.org/10.1002/mrm.26886

55. Ma D, Jones SE, Deshmane A, Sakaie K, Pierre EY, Larvie M, McGivney D, Blümcke I, Krishnan B, Lowe M, Gulani V, Najm I, Griswold MA, Wang ZI (2019) Development of high-resolution 3D MR fingerprinting for detection and characterization of epileptic lesions. J Magn Reson Imaging 49:1333-1346. https://doi.org/10. 1002/jmri.26319 
56. Does MD (2018) Inferring brain tissue composition and microstructure via MR relaxometry. Neuroimage 182:136-148. https://doi. org/10.1016/j.neuroimage.2017.12.087

57. Nilsson M, Englund E, Szczepankiewicz F, van Westen D, Sundgren PC (2018) Imaging brain tumour microstructure. NeuroImage 182:232-250. https://doi.org/10.1016/j.neuroimage. 2018.04.075

58. Fujita S, Buonincontri G, Cencini M, Fukunaga I, Takei N, Schulte RF, Hagiwara A, Uchida W, Hori M, Kamagata K, Abe O, Aoki S (2020) Repeatability and reproducibility of human brain morphometry using three-dimensional magnetic resonance fingerprinting. Hum Brain Mapp 42:275-285. https://doi.org/10.1002/hbm.25232

59. Buonincontri G, Biagi L, Retico A, Cecchi P, Cosottini M, Gallagher FA, Gómez PA, Graves MJ, McLean MA, Riemer F, Schulte RF, Tosetti M, Zaccagna F, Kaggie JD (2019) Multi-site repeatability and reproducibility of MR fingerprinting of the healthy brain at 1.5 and $3.0 \mathrm{~T}$. NeuroImage 195:362-372. https:// doi.org/10.1016/j.neuroimage.2019.03.047

60. Menze BH, Jakab A, Bauer S, Kalpathy-Cramer J, Farahani K, Kirby J, Burren Y, Porz N, Slotboom J, Wiest R, Lanczi L, Gerstner E, Weber MA, Arbel T, Avants BB, Ayache N, Buendia P, Collins DL, Cordier N, Corso JJ, Criminisi A, Das T, Delingette H, Demiralp C, Durst CR, Dojat M, Doyle S, Festa J, Forbes F, Geremia E, Glocker B, Golland P, Guo X, Hamamci A, Iftekharuddin KM, Jena R, John NM, Konukoglu E, Lashkari D, Mariz JA, Meier R, Pereira S, Precup D, Price SJ, Raviv TR, Reza SMS, Ryan M, Sarikaya D, Schwartz L, Shin HC, Shotton J, Silva CA, Sousa N, Subbanna NK, Szekely G, Taylor TJ, Thomas OM,
Tustison NJ, Unal G, Vasseur F, Wintermark M, Ye DH, Zhao L, Zhao B, Zikic D, Prastawa M, Reyes M, van Leemput K (2015) The multimodal brain tumor image segmentation benchmark (BRATS). IEEE Trans Med Imaging 34:1993-2024. https://oi.org/10.1109/ TMI.2014.2377694

61. Kofler F, Berger C, Waldmannstetter D, Lipkova J, Ezhov I, Tetteh G, Kirschke J, Zimmer C, Wiestler B, Menze BH (2020) BraTS Toolkit: translating BraTS brain tumor segmentation algorithms into clinical and scientific practice. Front Neurosci 14. https://doi. org/10.3389/fnins.2020.00125

62. Berglund J, van Niekerk A, Rydén H, Sprenger T, Avventi E, Norbeck O, Glimberg SL, Olesen OV, Skare S (2021) Prospective motion correction for diffusion weighted EPI of the brain using an optical markerless tracker. Magn Reson Med 85: 1427-1440. https://doi.org/10.1002/mrm.28524

63. Serrao EM, Kessler DA, Carmo B et al (2020) Magnetic resonance fingerprinting of the pancreas at $1.5 \mathrm{~T}$ and 3.0 T. Sci Rep 10:17563. https://doi.org/10.1038/s41598-020-74462-6

64. Sushentsev N, Kaggie JD, Buonincontri G, Schulte RF, Graves MJ, Gnanapragasam VJ, Barrett T (2020) The effect of gadoliniumbased contrast agent administration on magnetic resonance fingerprinting-based $\mathrm{T} 1$ relaxometry in patients with prostate cancer. Sci Rep 10:20475. https://doi.org/10.1038/s41598-020-773314

Publisher's note Springer Nature remains neutral with regard to jurisdictional claims in published maps and institutional affiliations. 